\section{Pacific Northwest}

National Laboratory

Operated by Battelle for the

U.S. Department of Energy

\title{
DCE Bio Detection System Final Report
}

\author{
MA Lind \\ GC Dunham \\ CR Batishko \\ MG Warner \\ GP Morgen \\ JA Willett
}

SL Owsley Jr

December 2007

Prepared for the U.S. Department of Energy under Contract DE-AC05-76RL01830 


\title{
DISCLAIMER
}

This report was prepared as an account of work sponsored by an agency of the United States Government. Neither the United States Government nor any agency thereof, nor Battelle Memorial Institute, nor any of their employees, makes any warranty, express or implied, or assumes any legal liability or responsibility for the accuracy, completeness, or usefulness of any information, apparatus, product, or process disclosed, or represents that its use would not infringe privately owned rights. Reference herein to any specific commercial product, process, or service by trade name, trademark, manufacturer, or otherwise does not necessarily constitute or imply its endorsement, recommendation, or favoring by the United States Government or any agency thereof, or Battelle Memorial Institute. The views and opinions of authors expressed herein do not necessarily state or reflect those of the United States Government or any agency thereof.

\author{
PACIFIC NORTHWEST NATIONAL LABORATORY \\ operated by \\ BATTELLE \\ for the \\ UNITED STATES DEPARTMENT OF ENERGY \\ under Contract DE-AC05-76RL01830
}

Printed in the United States of America
Available to DOE and DOE contractors from the Office of Scientific and Technical Information,
P.O. Box 62, Oak Ridge, TN 37831-0062;
ph: (865) 576-8401
fax: $(865)$ 576-5728
email: reports@adonis.osti.gov

\begin{abstract}
Available to the public from the National Technical Information Service, U.S. Department of Commerce, 5285 Port Royal Rd., Springfield, VA 22161 ph: (800) 553-6847 fax: $(703) 605-6900$ email: orders@ntis.fedworld.gov online ordering: http://www.ntis.gov/ordering.htm
\end{abstract}

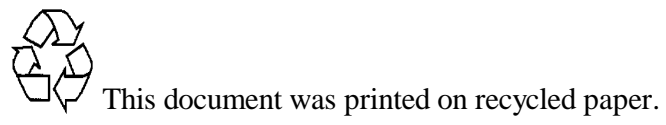




\title{
DCE Bio Detection System Final Report
}

\author{
Michael Lind \\ Charles Batishko \\ Gerry Morgen \\ Stan Owsley \\ Glen Dunham \\ Marvin Warner \\ Jesse Willett
}

December 31, 2007

Prepared for the USAF/Brooks City Base under MIPR AFMC277 No.RAF27704670060 with the U.S. Department of Energy under contract DE-AC05-76RL01830

Pacific Northwest National Laboratory

Richland, WA 99352 


\section{Table of Contents}

Introduction

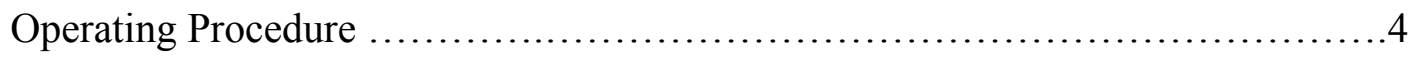

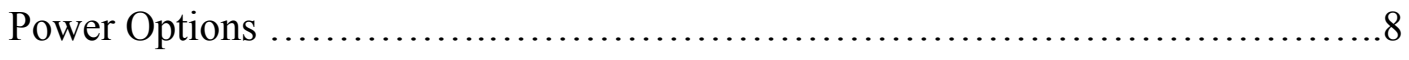

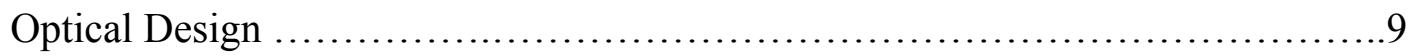

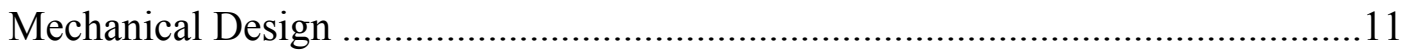

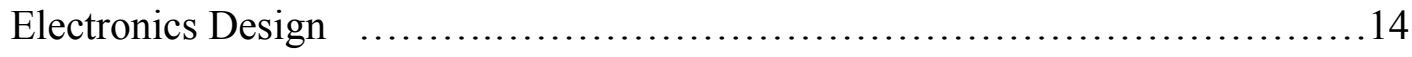

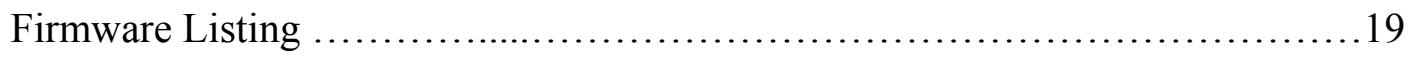

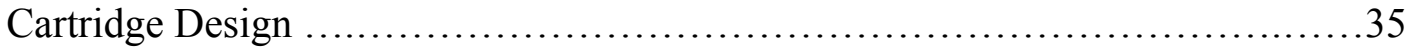




\section{List of Figures}

Figure 1. DCE Bio-Detection System showing the measurement module and a package of vacuum sealed cartridges. 2

Figure 2. Cartridge in measurement unit prior to lid closure. ..............................

Figure 3. Top view of the DCE measurement apparatus showing the injection port, the LCD status display, the four LED level indicators and the four control buttons on the top side of the measurement instrument.

Figure 4. Optical block diagram for DCE Bio-Detection System. .......................9

Figure 5. Dimensioned drawing of the DCE instrument case. ...............................11

Figure 6. Bottom view of DCE instrument showing battery compartment. 12

Figure 7. Dimensional drawing of DCE flip top cartridge cover. .......................12

Figure 8. Dimensional drawing of DCE cuvette holder. ......................................13

Figure 9. Installed cuvette holder with adjacent light shield. ..............................13

Figure 10. DCE electronics wiring diagram. .......................................................14

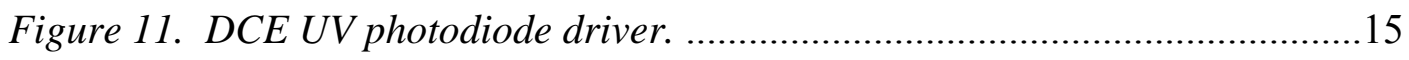

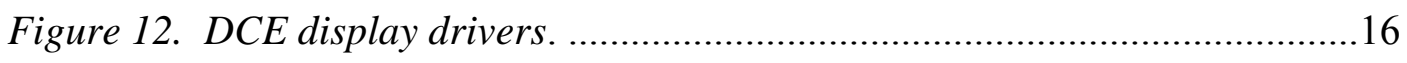

Figure 13. DCE optical signal amplifier schematics. …….................................17

Figure 14. DCE microprocessor controller schematic. ………………................18

Figure 15. Photographs of assembled DCE cartridge including close up of magnet and injection septum. .35

Figure 16. Dimensional drawing of DCE cartridge assembly. ..............................36

Figure 17. Dimensional drawing of DCE cartridge molded top detail. .37

Figure 18. DCE cartridge bottom detail. 38 


\section{Introduction}

The DNA Capture Element (DCE) Bio-

Detection System (Biohound) was

conceived, designed, built and tested by

PNNL under a MIPR for the US Air Force

under the technical direction of Dr.

Johnathan Kiel ${ }^{1}$ and his team at Brooks City

Base in San Antonio Texas. The project was

directed toward building a measurement

device to take advantage of a unique

aptamer based assay developed by the Air

Force for detecting biological agents. The

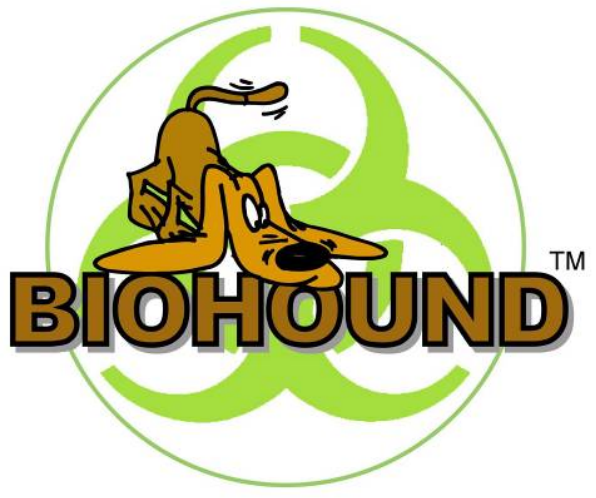

assay uses narrow band quantum dots fluorophores, high efficiency fluorescence

quenchers, magnetic micro-beads beads and selected aptamers to perform high

specificity, high sensitivity detection of targeted biological materials in minutes. This

final report summarizes and documents the final configuration of the system delivered to the Air Force in December 2008.

While the details of the assay will not be discussed here, the basic detection process is simple in concept. Detection aptamers (short single strand DNA segments) are formulated for specific target molecules and attached to functionalized quantum dot fluorophores along with an appropriate spaced non-radiative quencher. In the absence of a complimentary DNA fragment supplied by the target biological, the proximity of the quencher and the quantum dot prevent the quantum dot from fluorescing. When a complimentary DNA fragment attaches itself to the aptamer with the fluorophore, the physical separation of the quencher and the quantum dot changes sufficiently to allow fluorescence in the presence of a suitable excitation source. Thus with no target molecule present, the solution remains dark. If a target is present, the solution glows when illuminated by the proper light source. If there is sufficient target present one can see the glow visually. If only trace quantities are present it requires a sensitive detector and a concentrator to see the fluorescence. The DCE Bio-Detection System described in this report fills that trace detection function.

The emitted light indicates the presence of the target molecule and the number of photons detected is proportional to the concentration of the target, the number of binding sites on the target, the efficiency of binding, the efficiency of de-quenching, the temperature, the optical obscuration fraction and a host of other factors. So while the biological sensitivity is target specific, the instrument sensitivity is more easily calibrated as a function of the minimum number of detectable quantum dots. For this DCE instrument the nominal detection noise threshold is estimated at 5-10 femto moles of quantum dots. The correlation to the biological target threshold has not yet been determined.

\footnotetext{
${ }^{1}$ AFMC277 No.RAF27704670060 dated 18 April 2004
} 
A key to high sensitivity detection is concentration of the target. In this DCE instrument, concentration is achieved using magnetic micro beads with affinity binders attached. There are many variations of this scheme possible. In one simple variation the beads act as collectors for classes of organic molecules. When put into a buffer solution and sprayed on an area to be analyzed, they will preferentially attract the biological materials of interest essentially magnetizing those materials. When the sprayed area is sampled using any of a number of standard sample collection methods, mixed with the aptamer/quantum dot solution, and subsequently flowed past a strong magnet, the magnetic beads are attracted to and localized by that magnet for detection. This magnetic concentration occurs in the DCE cartridge where volumetric concentration approaching 1000 can be achieved.

The DCE Detection System incorporates both the optics/electronics and the collection cartridge into a single measurement system. Figure 1 is a photograph of the complete measurement system showing the detection unit and a vacuum sealed package containing four measurement cartridges.

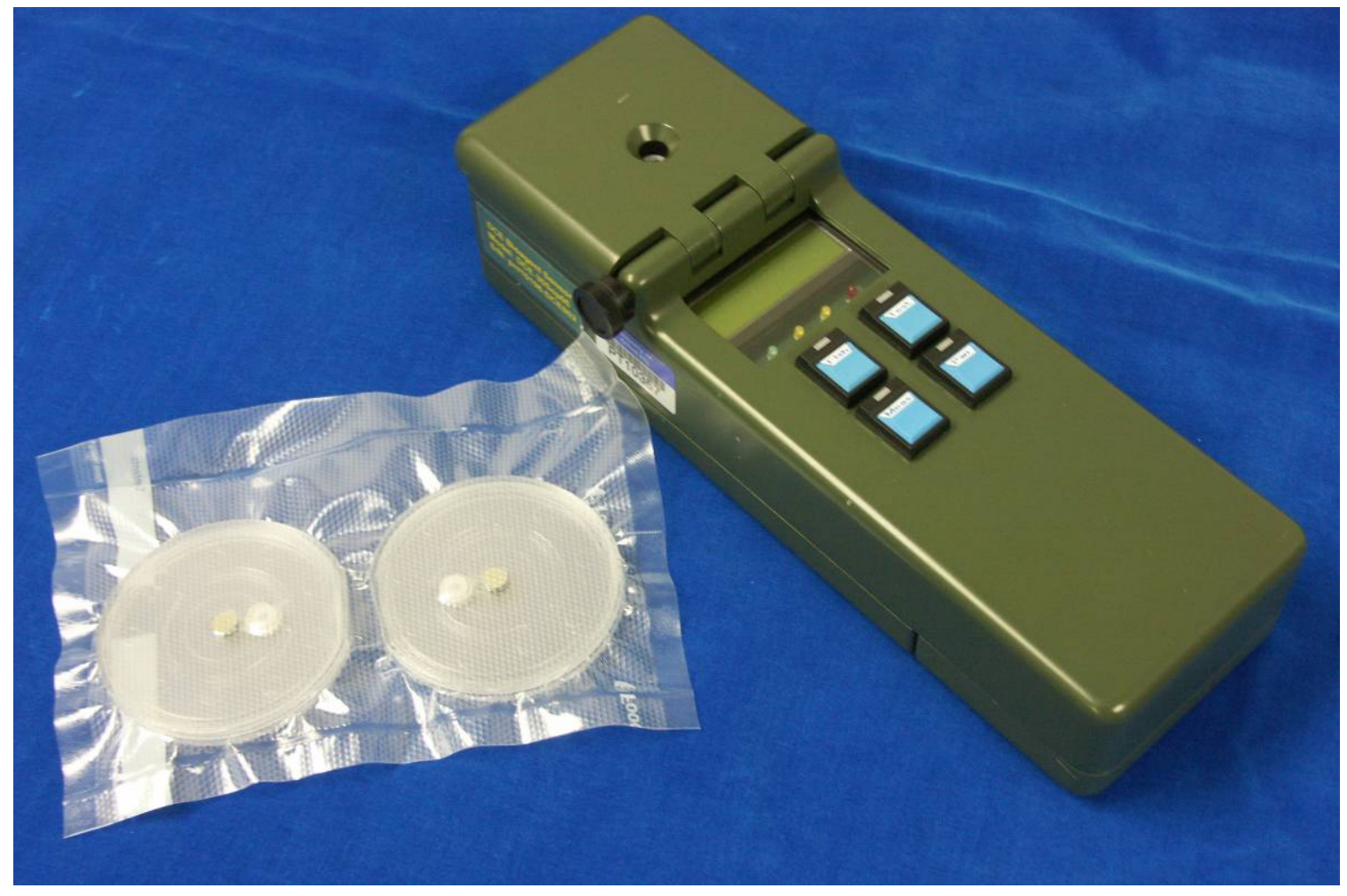

Figure 1. DCE Bio-Detection System showing the measurement module and a package of vacuum sealed cartridges.

The DCE Bio-Detection System while shown in the preferred mode in Figure 1 supports several other measurement modes. One alternative uses a glass plate that has a $5 \mu 1$ well to hold a $5 \mu \mathrm{l}$ sample that is covered with a microscope slip cover. Another alternative uses a second specially designed flip top lid that houses a removable rectangular cuvette centered over the detector port. Using this method, solutions can be put into a cuvette for 
incubation, placed over a magnet to concentrate any magnetic particles at the bottom of the cuvette. The cuvette is then placed into the special lid which places the bottom of the cuvette over the measurement area and a light tight cover is placed over the cuvette. This allows the measurement of the contents at the bottom of the cuvette only. The preferred method uses a flow cell cartridge that draws in a solution from a syringe via an injection septum. The cartridges have a small integral magnet attached over the measurement area that attracts magnetic particles to concentrate them over the measurement port. The cartridge has the advantage of being a sealed system that can be externally decontaminated, retained for subsequent forensic analysis, and is inherently chain of custody friendly.

There are many possible extensions to this prototype measurement system. In principle the system can be used for any type of sandwich assay employing an affinity binder a fluorophore reporter (dye or quantum dot) and a magnetic particle. For example it has been used successfully on a botulinum toxin assay using antibodies, dyes and magnetic nano-particles. The device could also be extended to multiple simultaneous assays with changes to the optical package and multiple color reporters. 


\section{Operating Procedure}

The DCE Bio-Detection System is designed to be easy to operate with limited training and minimal manual dexterity. The measurement cartridges may be loaded by syringe injection through its vacuum septum outside of the measurement unit or inside the measurement unit. The cartridge is inserted into the measurement unit by lifting the hinged cover and placing it with the magnet side up in the fitted recess. Closing the cover provides optical background isolation for maximum sensitivity. Figure 2 shows a cartridge mounted in the proper configuration.

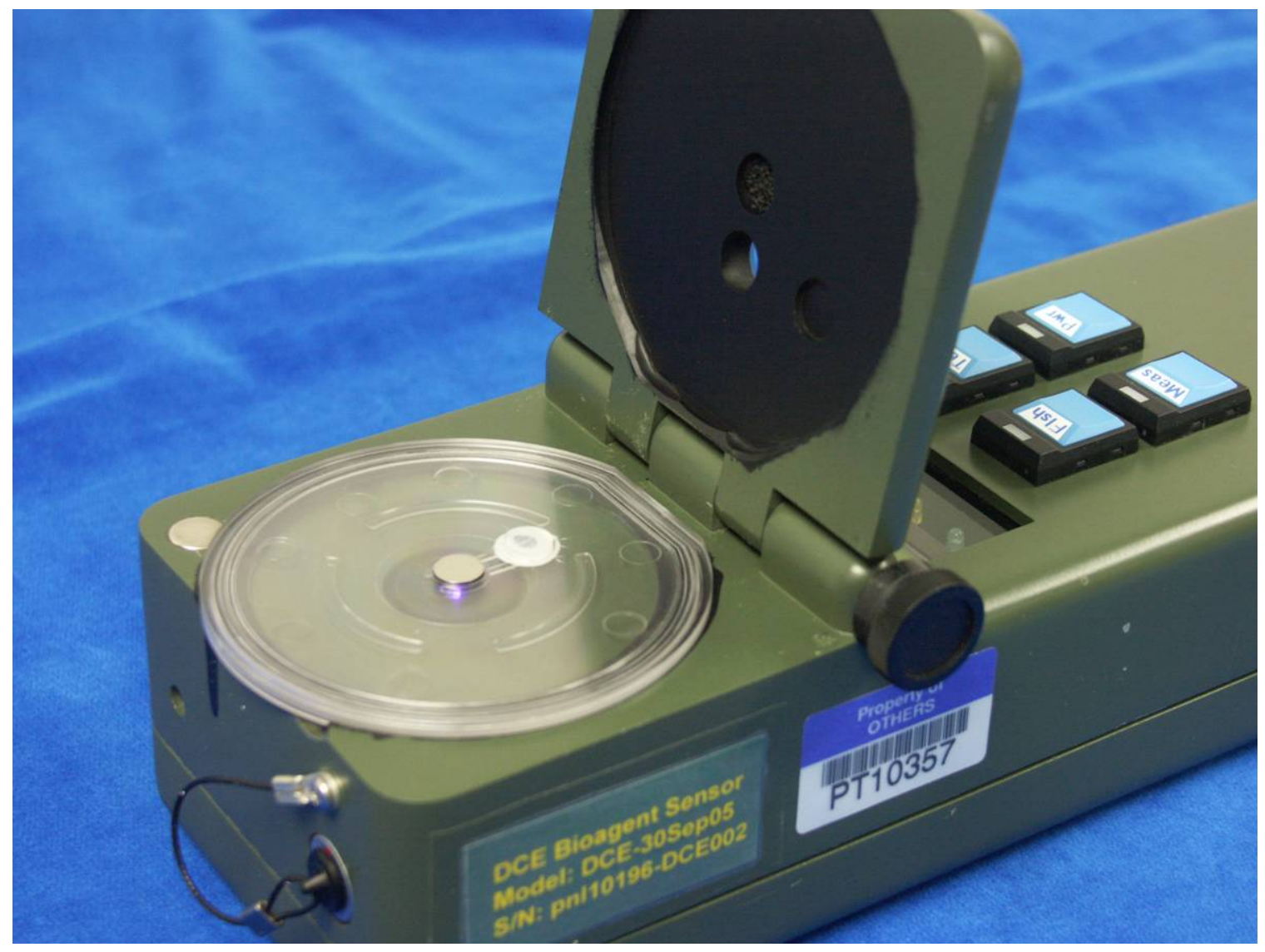

Figure 2. Cartridge in measurement unit prior to lid closure.

There are four momentary pushbutton switches that control the actual measurement sequence., one for turning on and off the power, and three function or mode switches. There is also a liquid crystal alphanumeric display for indicating system status and four LEDs which indicate the relative level of target agent detected. These switches and displays are shown in the Figure 3 below. 


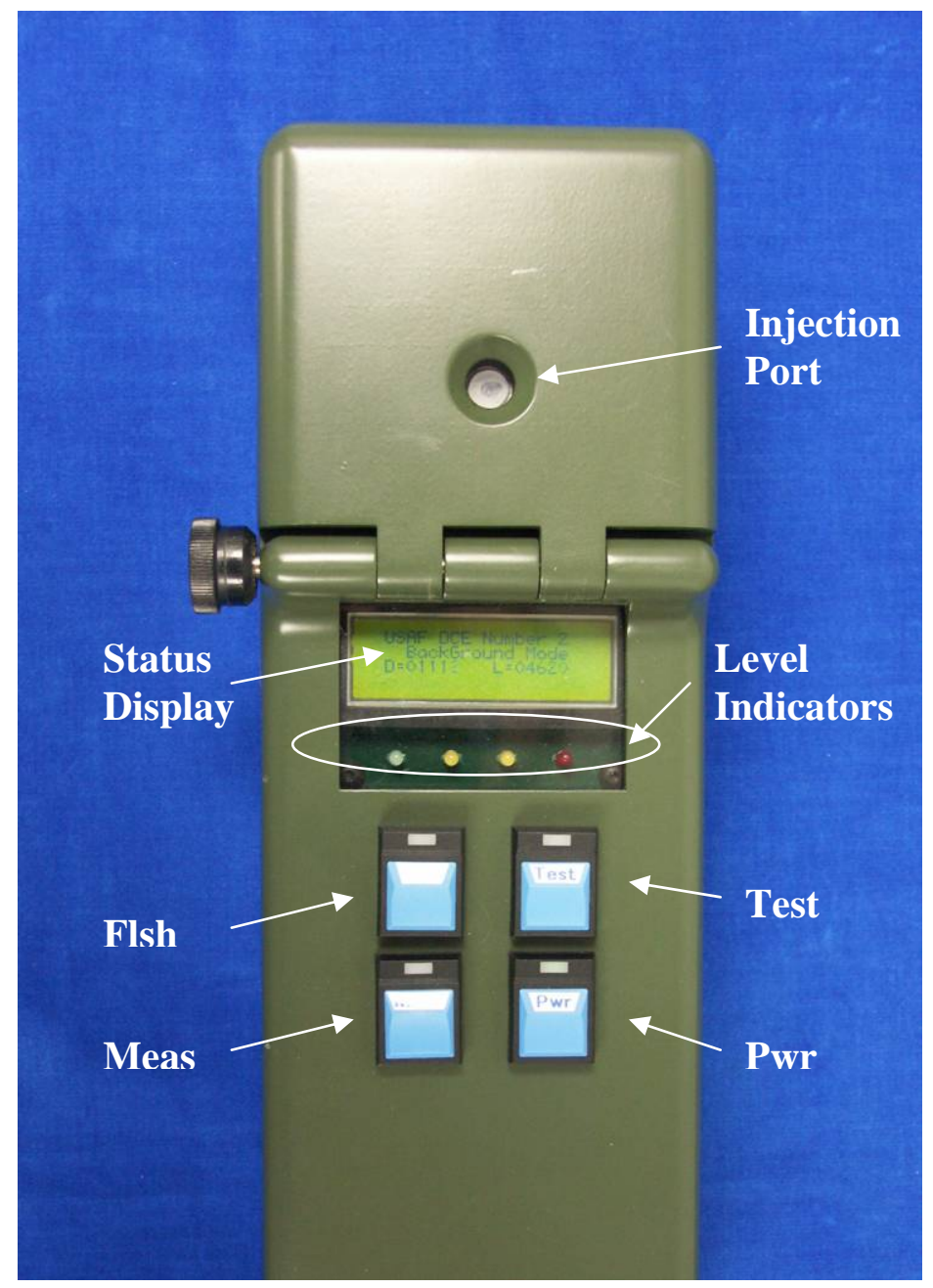

Figure 3. Top view of the DCE measurement apparatus showing the injection port, the $L C D$ status display, the four LED level indicators and the four control buttons on the top side of the measurement instrument.

The switches are labeled:

Pwr - Power On / Off Switch (lower right)

Meas - Measure Mode (lower left)

Test - Test Mode (upper right)

FIsh - Flash Light Mode (upper left)

After applying power, either using batteries or the laboratory power supply, the DCE is turned on by pressing the lower right $\boldsymbol{P} w \boldsymbol{r}$ switch for 1 to 2 seconds. The red LED in the lower left Meas switch will blink on then off during the initialization process which indicates the instrument is functioning properly and has entered the startup mode. Once the DCE is initialized and the power latched, the LCD displays the startup banner. 
After a 5 second delay, the background mode displays raw data values. The data displayed is for the direct detector signal, D, the loc-in amplifier signal, L. The lock-in amplifier signal is the rectified AC component of the fluorescence signal that is synchronously modulated with the illumination source, rejecting much of the asynchronous background noise. Although L is the primary signal of interest, D provides a diagnostic indication of electronics malfunction, DC drift, stray light problems and detector saturation problems Normal values in the background mode should look something like $\mathrm{D}=00548 \mathrm{~L}=02370$. These numbers derived from a 16-bit analog to digital converter (ADC) which has a range 0 - 65535 (0 to 2.048 volts.

The background mode displays during the warm up period. Depending on environmental conditions, up to 20 minutes warm-up is required to obtain low drift readings after the device is first powered up. It is advisable to place an unused cartridge in the measurement apparatus to obtain the most representative background (null sample) readings.

Pressing and releasing the lower left Meas switch again transitions from the background mode to the measure mode saving the background readings to be used as an offsets. The background readings are subtracted from the new $\mathrm{D}$ and $\mathrm{L}$ values being acquired with the filled cartridge. Simultaneously the four indicator light emitting diodes (LEDs) [Green, Yellow, Yellow, and Red] will activate. These LEDs are used to indicate a range of D and $\mathrm{L}$ values. The ranges are listed below and were selected based upon quantum dot calibration data.

Green: $\quad \mathrm{L}=0$ to 199

Yellow: $\quad \mathrm{L}=200$ to 599

Both Yellow: $\mathrm{L}=600$ to 1999

Red: $\quad$ L $>2000$

Pressing and releasing the lower left Meas switch again transitions back to the background mode to prepare for another measurement cycle.

Pressing and releasing the upper right Test switch transitions from the background mode to the test mode. This mode is used for general testing and setup, such as checking flow cells or cuvettes for fluorescence levels before being used in a measurement procedure.

Remember that finger oils, cigarette smoke, and a host of other common contaminates are highly fluorescent and can significantly interfere with these sensitive measurements.

The test mode is similar to the measure mode, except the LEDs are not active. When going from background mode to test mode, the $\mathrm{D}$ and $\mathrm{L}$ values at the time the switch is pushed are used as an offset, and subtracted from the new D and L values being acquired. The values displayed can go negative if there is fluorescent material in the optics field of view when the Test switch is pressed and then removed during the Test mode. Pressing and releasing the Test switch again reverts back to the background mode. 
Note that the Meas and Test switches transition to the measure mode and test mode only from the background mode. If the Test switch is pressed when in the measure mode, DCE will go to the background mode and Test must be pressed again to transition to the test mode. The same operation holds for the Meas switch if in the test mode.

Pressing and releasing the upper left Flsh switch transitions to the flashlight mode. This turns off the UV LED used for measure mode and test mode and turns on a UV LED mounted in the end of the DCE box - the same end as the DC power plug. The Meas and Test switches do not function in the flash light mode. This mode could be used to search for areas or objects that fluoresce. Pressing the Flsh switch again transitions out of flash light mode and reverts to background mode. 


\section{Power Options}

The unit can be powered by batteries for remote operation or by an external laboratory DC power supply for doing extended time frame measurements in the laboratory. The laboratory power supply provided is model Instek GPS-4303.

Battery operation uses three 9 volt batteries. Lithium batteries are preferred for their extended capacity and wider temperature capabilities, but standard alkaline cells will work. To install the batteries, remove the small cover at the lower end of the rear of the case and insert them into the holders.

Batteries can remain in the unit when using the external laboratory power supply. The instrument detects when the external power source is being used and draws from the external source. When the battery only is present, the instrument will automatically turn off after 15 minutes if inactivity (i.e. no switches have been pushed). If the external power supply is being used and the batteries are installed, the instrument will automatically switch to battery operation if the external power source is removed without turning the unit off. This allows for extended warm up periods prior to mobile use.

The laboratory supply is used to generate +10.0 volts $\&-10.0$ volts for the electronics, and a separate 10.0 volts for the UV LED operation. After turning on the power supply, check and set the following voltages:

$\mathrm{CH} 1=10.0$ volts

$\mathrm{CH} 2=10.0$ volts

$\mathrm{CH} 4=10.0$ volts $\quad$ [upper left slide switch toggles $\mathrm{CH} 2-\mathrm{CH} 4$ display]

A power cable with connectors, red/black banana plugs for connections to the lab power supply and 5 pin connector for the DCE box, is provided. The Blue-Black wire pair connects to $\mathrm{CH} 1$, with the red plug to the red terminal and the black plug to the black terminal. The White-Black wire pair connects to $\mathrm{CH} 2$, with the red plug to the red terminal and the black plug to the black terminal. The Red-Black wire pair connects to $\mathrm{CH} 4$, with the red plug to the red terminal and the black plug to the black terminal. Internal wiring determines which supply is positive and which is negative.

After turning on the Instek GPS-4303 POWER Switch, the OUTPUT button must be pushed to activate the power supply output terminals. When using the lab power supply, turn it on, check that the 3 output voltages are all 10.0 volts, turn on the output, then plug the DC power connector into the DCE. Turn on the DCE by pressing the DCE power switch $-\boldsymbol{P} w \boldsymbol{r}$. Turn off the DCE by again pressing the DCE power switch $-\boldsymbol{P} w \boldsymbol{r}$. 


\section{Optical Design}

The DCE is specifically designed to detect and measure the fluorescence of the Evident Technologies Fort Orange Qdots in a solution at a wavelength 600nm. All components were selected to maximize performance without regard to individual component cost. Maximum throughput and minimum out of band transmission, maximum stray light rejection, minimum component fluorescence, minimum polarization sensitivity and minimum internal scatter were the goals driving the design and selection of components. The simple optical system layout is shown in block form in Figure 4.

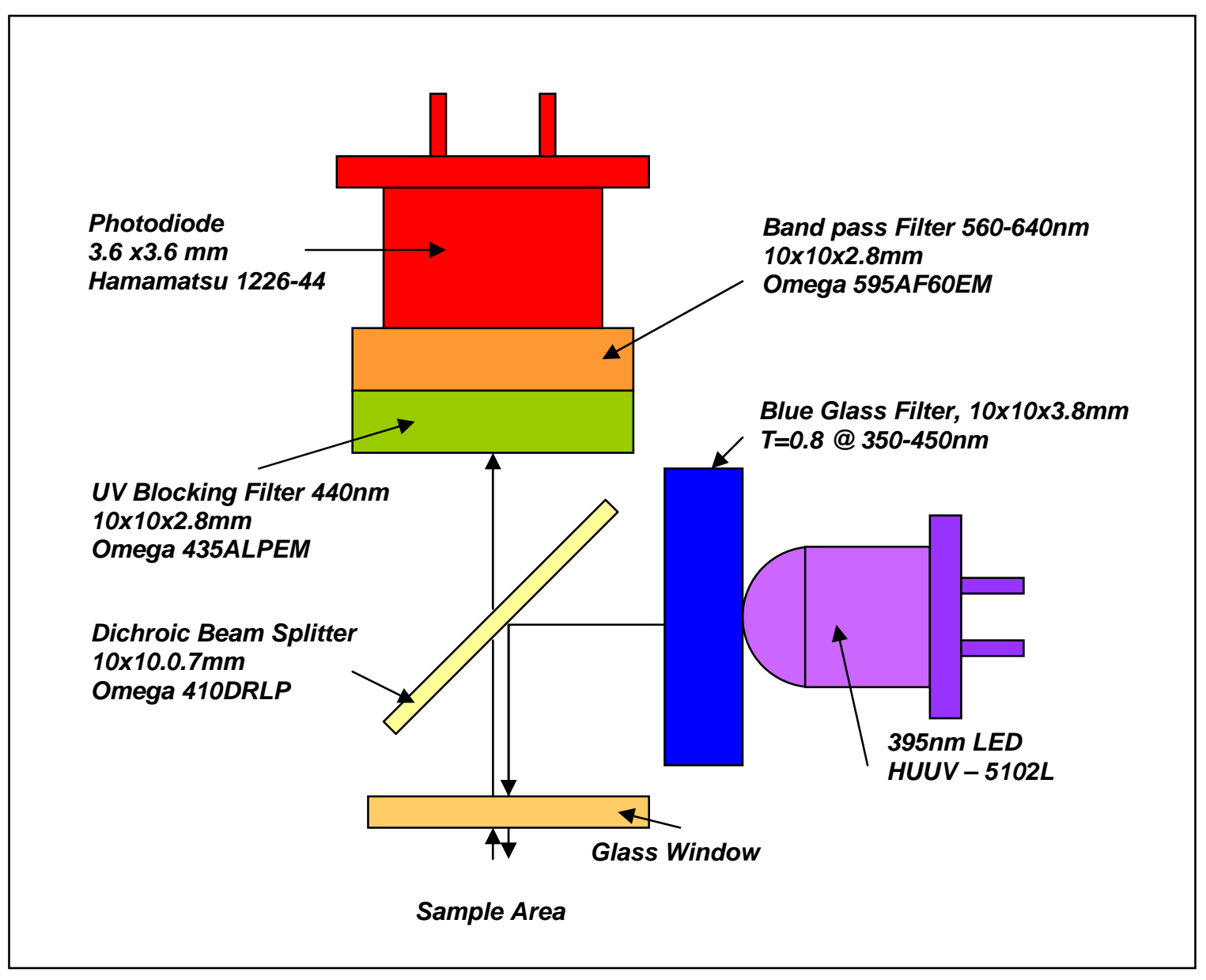

Figure 4. Optical block diagram for DCE Bio-Detection System.

The optical source to stimulate fluorescence is a UV LED that operates at $395 \mathrm{~nm}$. It is operated in pulsed mode running at $1 \mathrm{kHz}$ with a duty cycle of $12 \%$ in order to take advantage of the stray light rejection of lock-in detection. The output of the diode is filtered to remove long wavelength components from the source which will compromise the low level performance of the system. The $395 \mathrm{~nm}$ UV light which maximizes the fluorescence excitation of the dyes and quantum dots used in the assays is directed onto the sample area using a 45 degree dichroic beam splitter. The resulting diffuse downshifted fluorescence passes through the beam splitter and a series of blocking/band 
pass filters that are centered on the Fort Orange response of $600 \mathrm{~nm}$. The low pass filter absorbs reflected UV light above $430 \mathrm{~nm}$ that might be scattered from the cartridge or the carrier fluid. An adjacent band pass filter narrows the pass band to the maximum

fluorescence region $(580 \mathrm{~nm})$ in order to further reduce ambient light effects. A solid state Hamamatsu photodiode is the sensor selected for high sensitivity and a large active area. 


\section{Mechanical Design}

The mechanical design for the instrument is driven by the following considerations:

- The optics assembly is protected from stay light to the greatest extent possible

- An easily accessible battery compartment

- Simple operational controls for use in conditions of impaired dexterity

- Indexed sample cartridge holder easily assessable with impaired dexterity

- Visible yet clandestine displays

- Sealed for rapid decontamination via bleach solution emersion

- Light weight, single hand carry and operation

Figure 5 below is a dimensioned drawing of the DCE instrument with top and side views. The bottom cover is not shown, but fits in two pieces to cover the electronics and the battery compartment. The electronics/optics compartment is o-ring sealed.

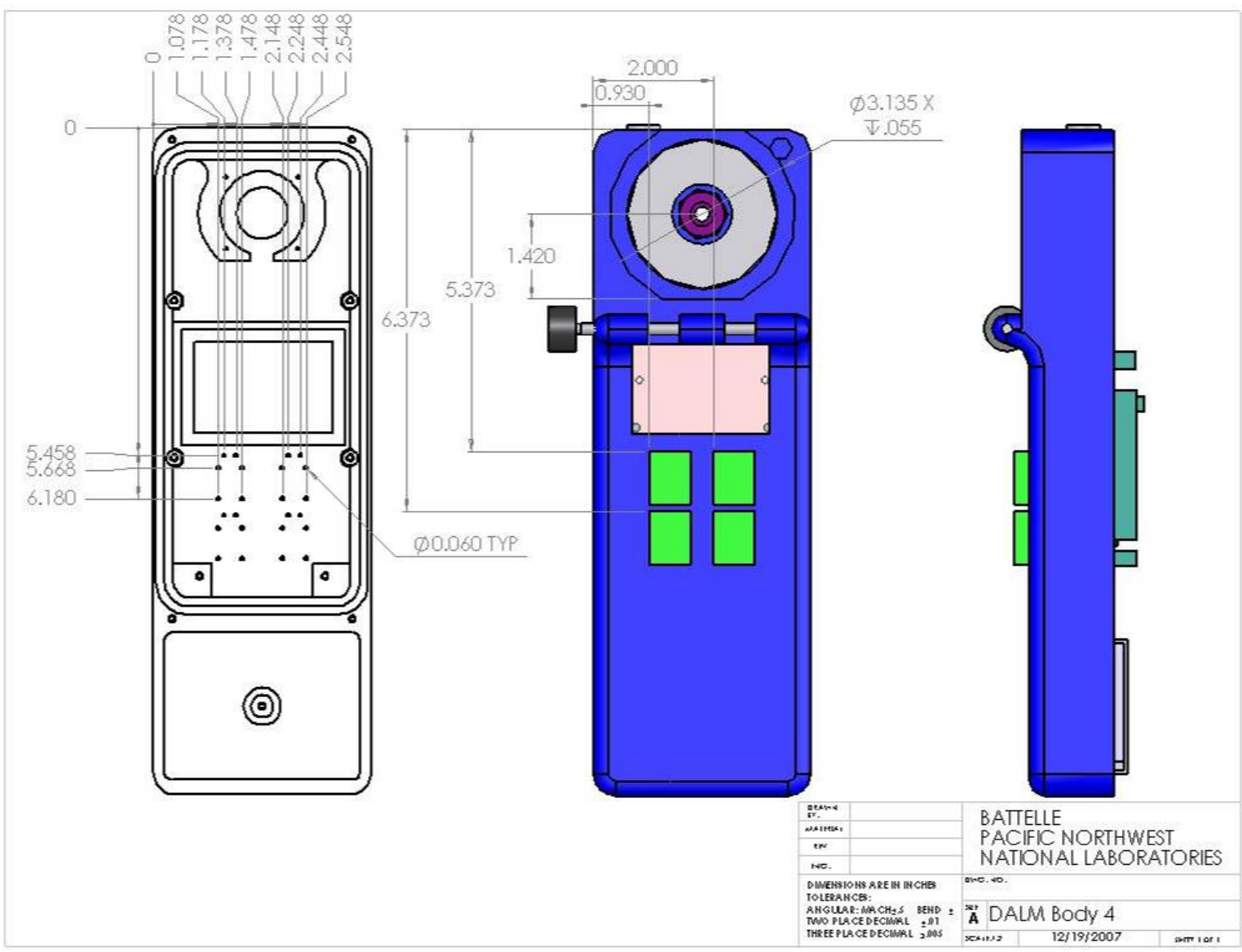

Figure 5. Dimensioned drawing of the DCE instrument case.

The bottom side of the instrument showing the battery compartment is shown in Figure 6 . 

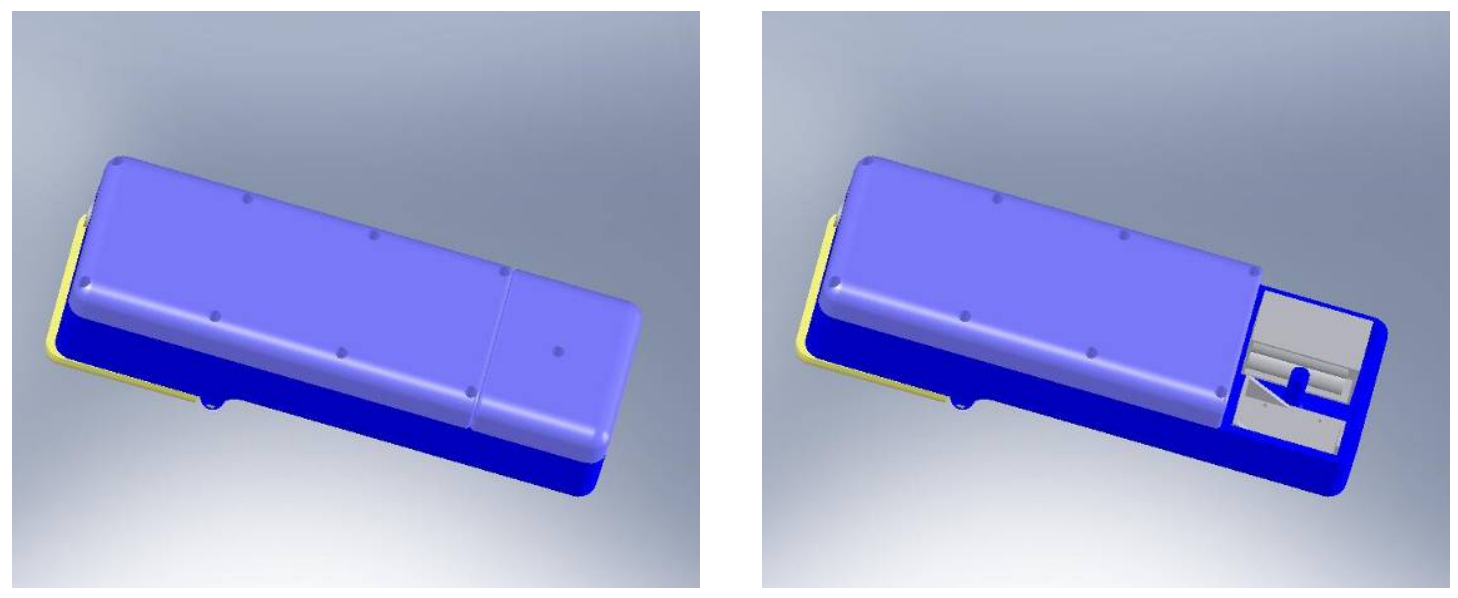

Figure 6. Bottom view of DCE instrument showing battery compartment.

Two flip top sample covers are supplied with the instrument. One is for covering the cartridge, the second is to hold a cuvette. The dimensional drawings of cartridge cover showing the fill port access is shown in Figure 7 and cuvette holder in Figure 8.

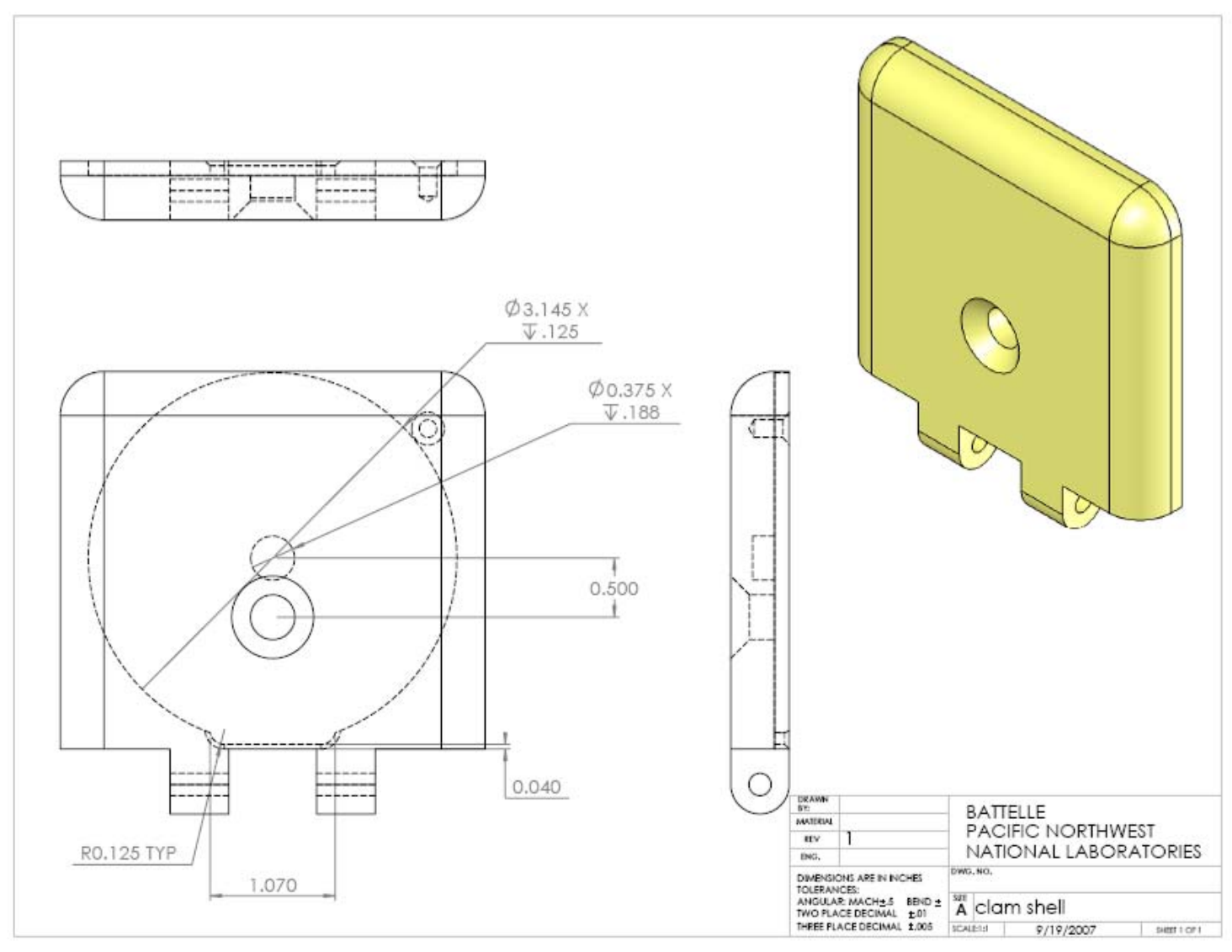

Figure 7. Dimensional drawing of DCE flip top cartridge cover 

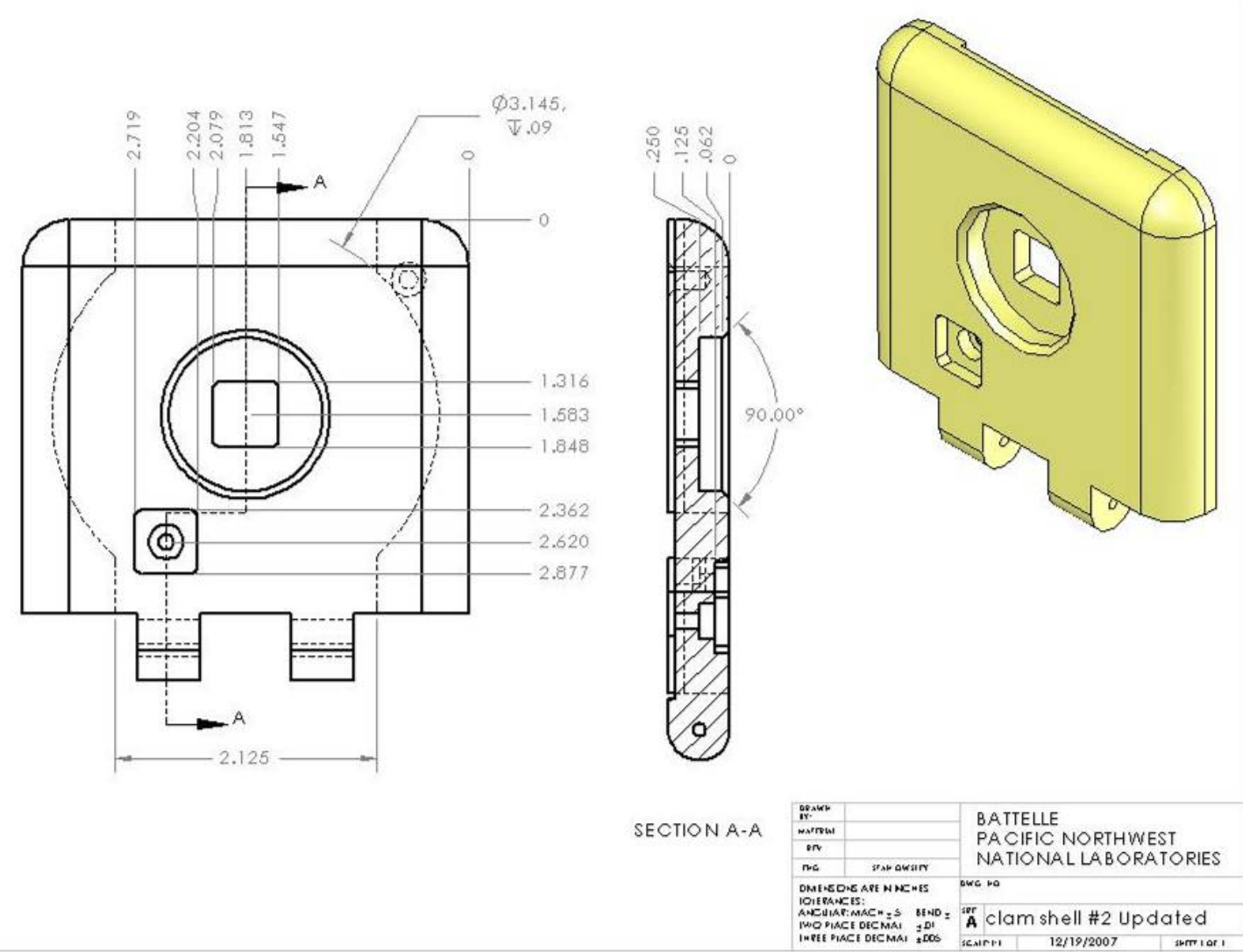

Figure 8. Dimensional drawing of DCE cuvette holder.

Figure 9 shows the cuvette holder installed on the DCE instrument. The cylinder is an accompanying light shield place over the cuvette after insertion.

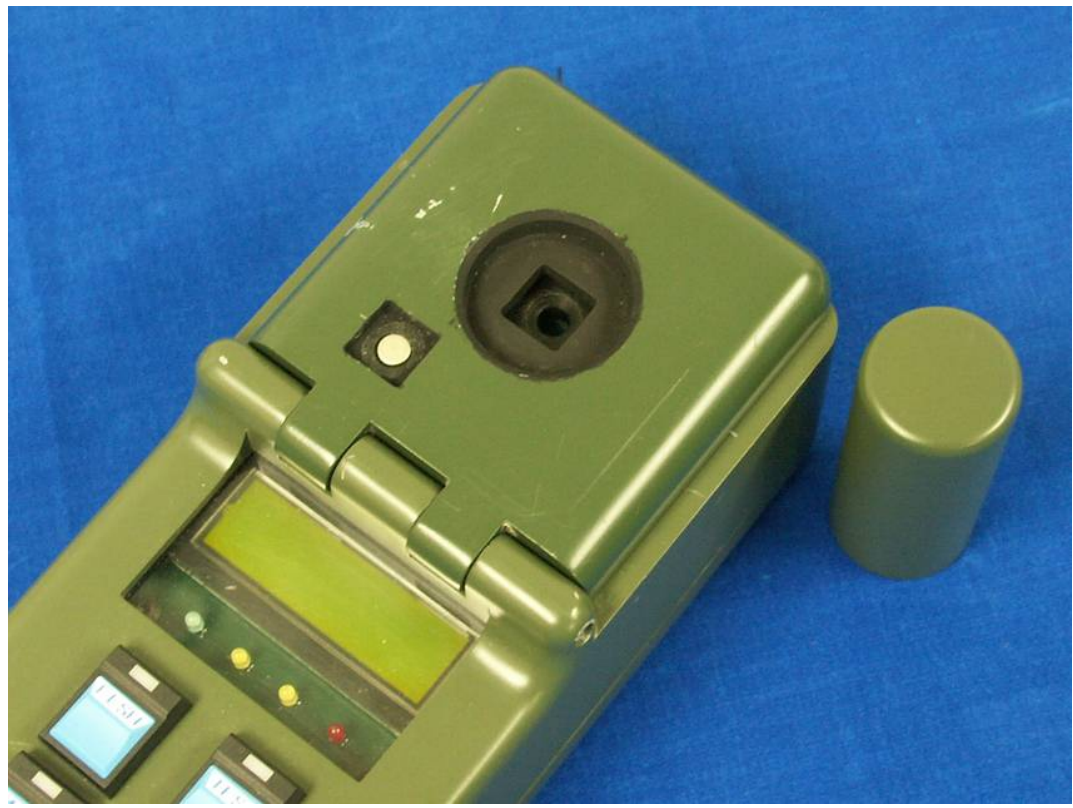

Figure 9. Installed cuvette holder with adjacent light shield. 


\section{Electronics Design}

The schematics for the electronics supporting the DCE instrument are shown in Figure 10-14. They represent the most recent red lined drawings for the delivered prototypes. All components are commercial off the shelf devices at the time the prototypes were assembled. Full sized drawing in $p d f$ files are available on the CD that accompanies this report.

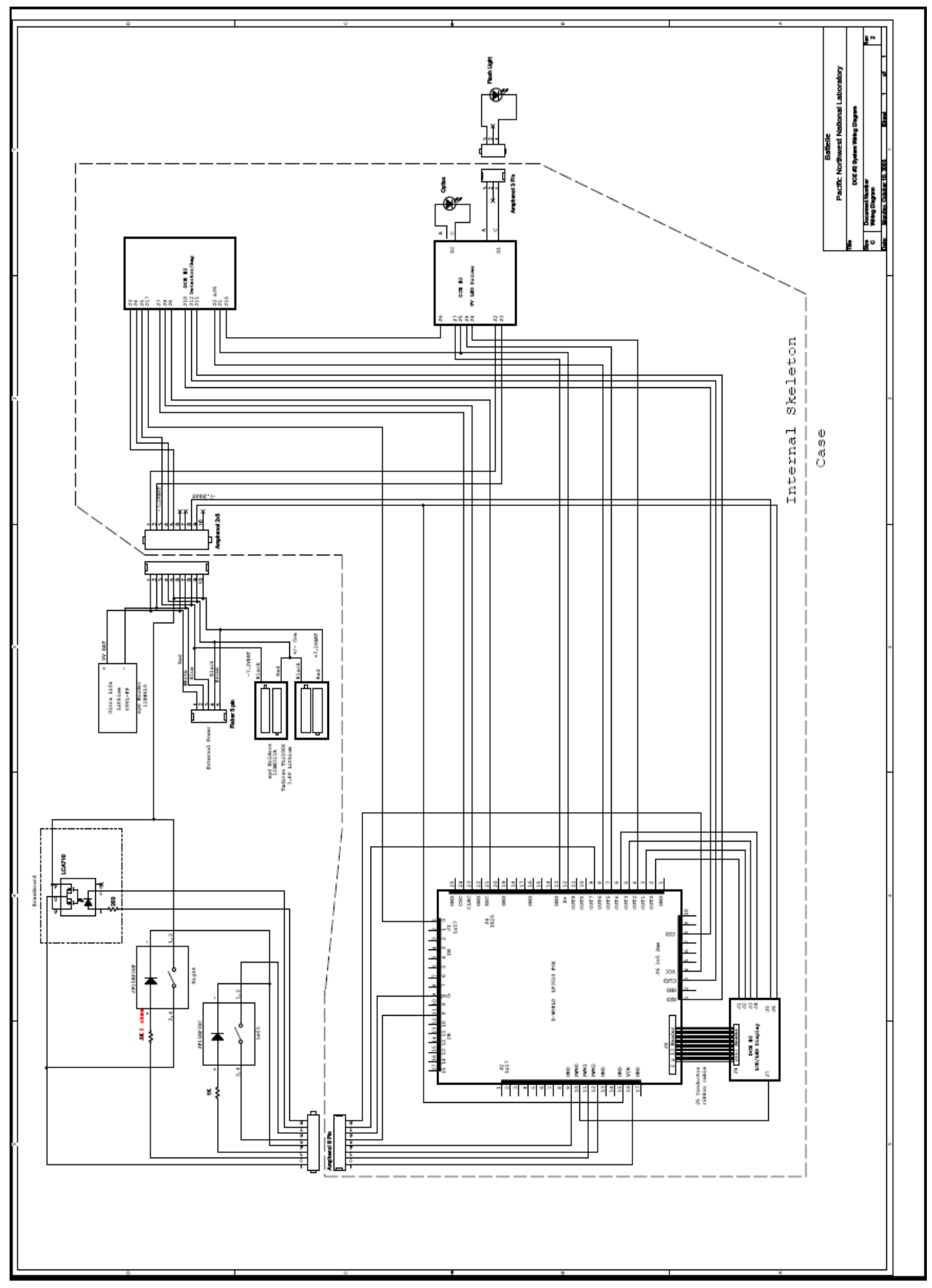

Figure 10. DCE electronics wiring diagram. 


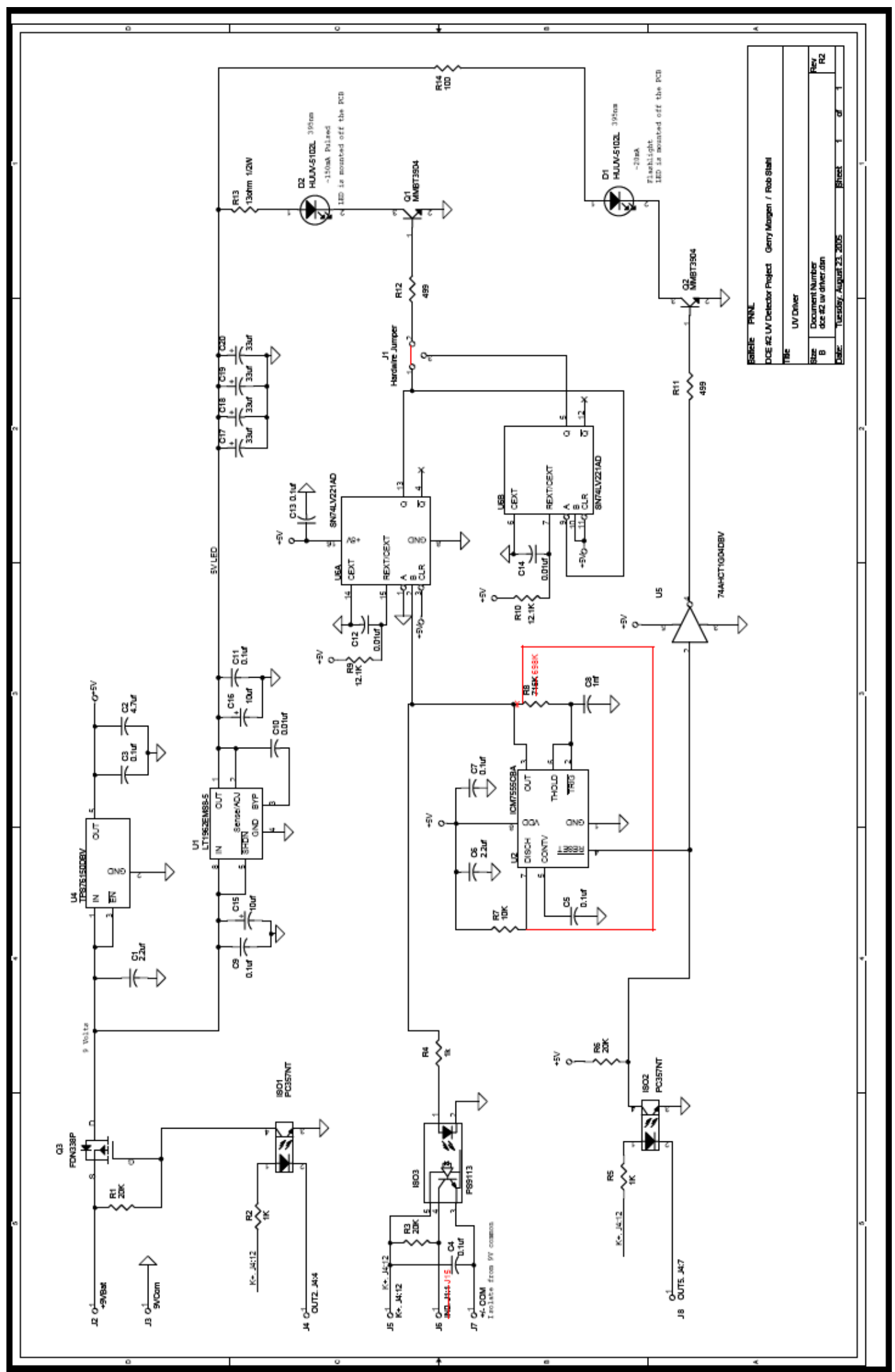

Figure 11. DCE UV photodiode driver. 


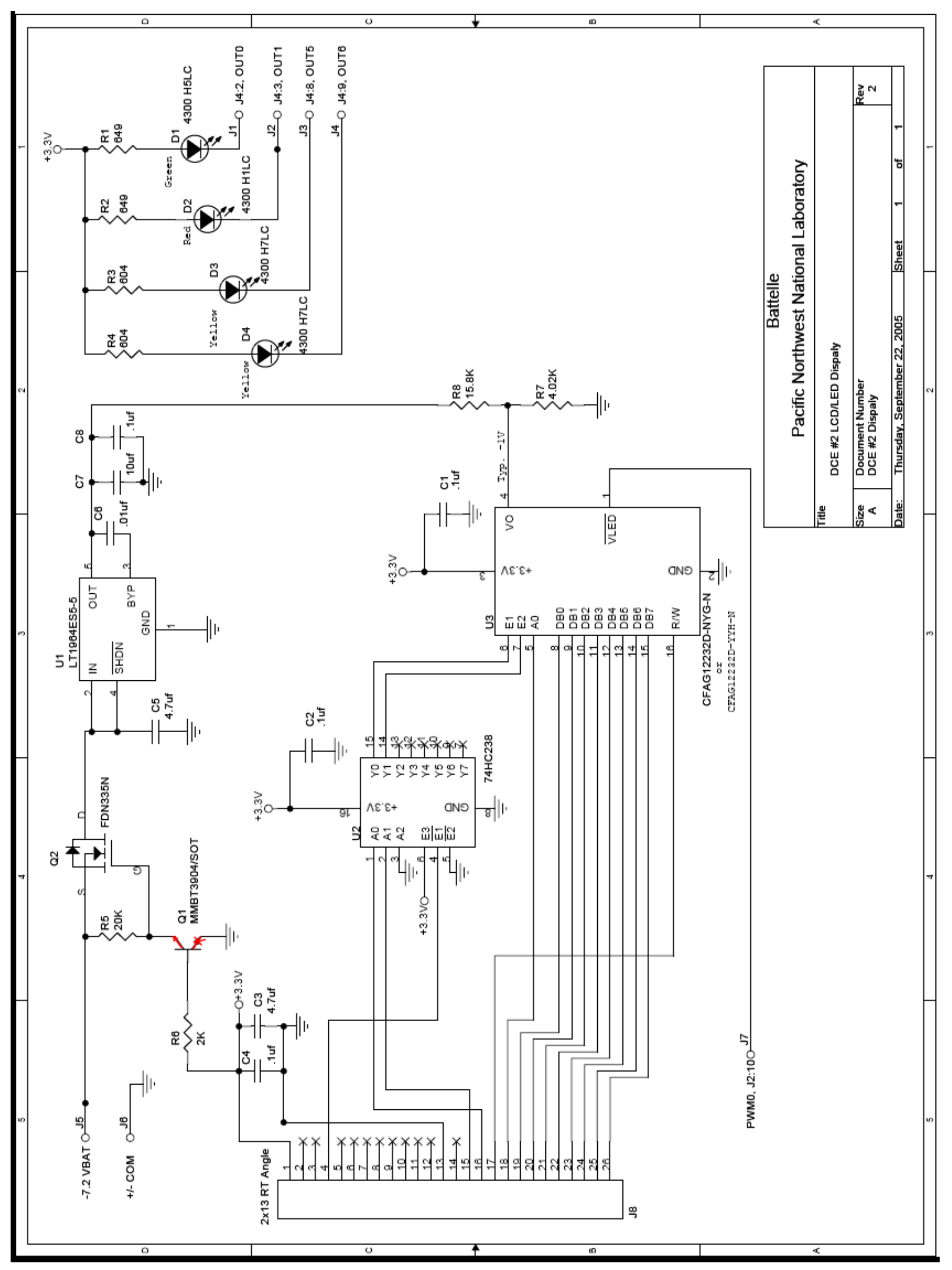

Figure 12. DCE display drivers. 


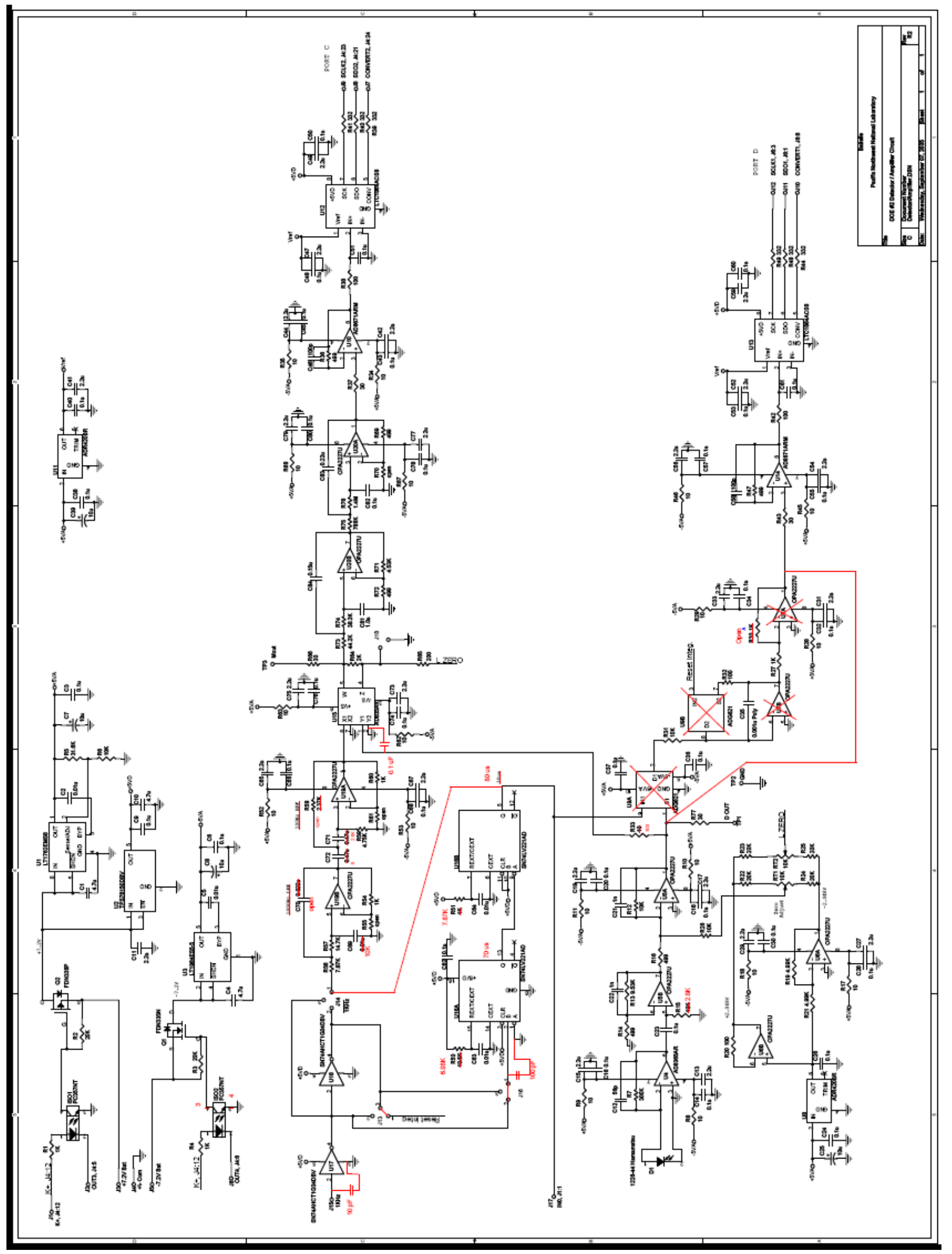

Figure 13. DCE optical signal amplifier schematics. 


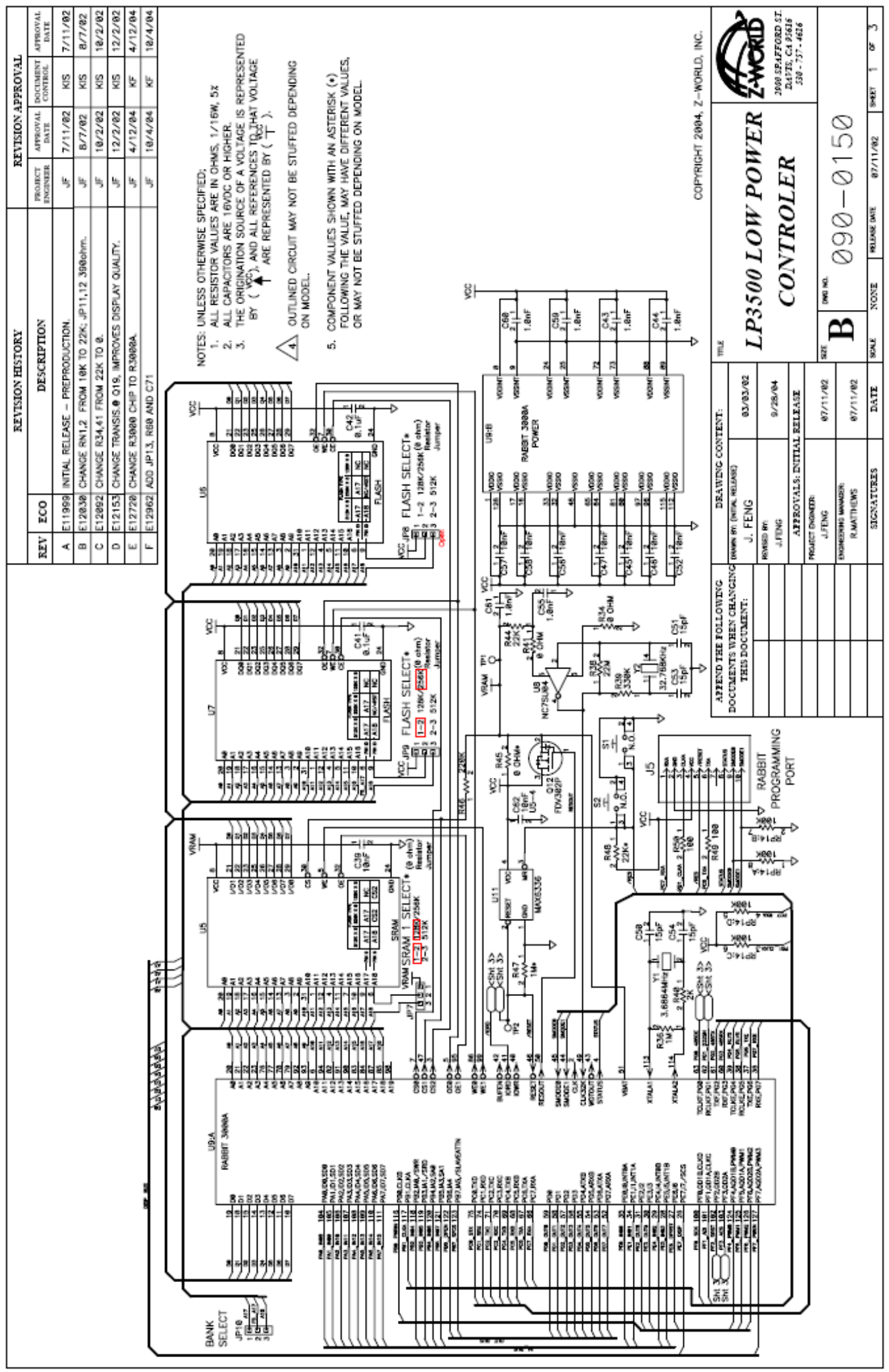

Figure 14. DCE microprocessor controller schematic. 


\section{Firmware Listing}

Notice: This computer software was prepared by Battelle Memorial Institute, hereinafter the Contractor, under Contract No. DE-AC05-76RL0 1830 with the Department of Energy (DOE). All rights in the computer software are reserved by DOE on behalf of the United States Government and the Contractor as provided in the Contract. You are authorized to use this computer software for Governmental purposes but it is not to be released or distributed to the public. NEITHER THE GOVERNMENT NOR THE CONTRACTOR MAKES ANY WARRANTY, EXPRESS OR IMPLIED, OR ASSUMES ANY LIABILITY FOR THE USE OF THIS SOFTWARE. This notice including this sentence must appear on any copies of this computer software.

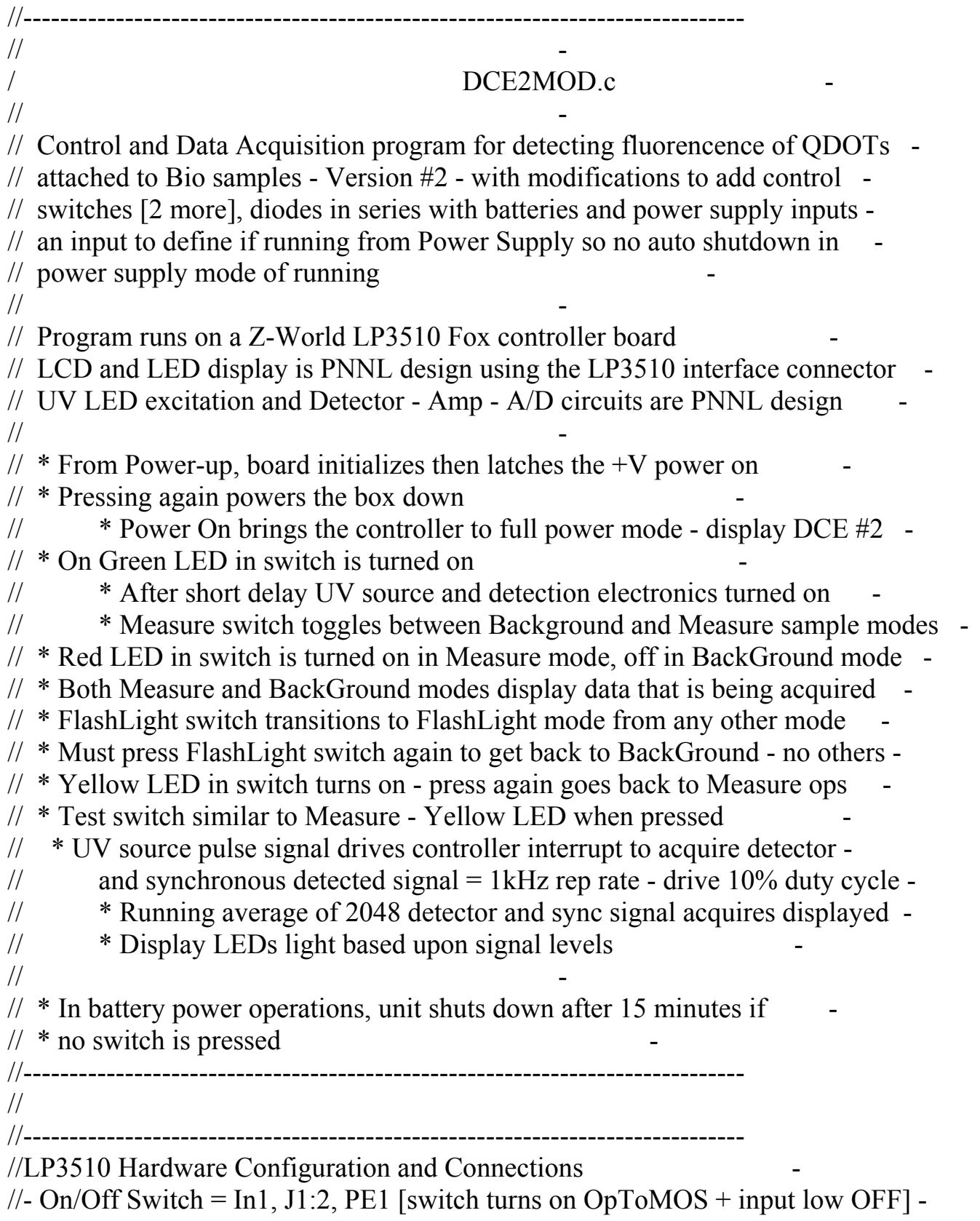


//- Measure Switch = In9, J1:11, PA1 [Vcc pullup switch takes to gnd] -

// - FlashLight Switch = IN10, J1:12, PA2 [Vcc pullup switch takes to gnd] -

$/ /$ - Test Switch = IN11, J1:13, PA3 [Vcc pullup switch takes to gnd] -

//- UV Drive Interrupt $=$ In0, J1:1, PE0 [positive edge triggered interrupt] -

$/ /$ - Power Source Input = In4, J1:5, PB2 [high = power supply - low = battery]-

// - Green Status LED = Out0, J4:2, PD0 [high out-FET on to gnd-LED on] -

// - Red Status LED = Out1, J4:3, PD1 [high out-FET on to gnd-LED on] -

// - UV LED Drive Power = Out2, J4:4, PD2 [high output turns on power] -

// - Yellow1 Status LED = Out3, J4:5, PD3 [high out-FET on to gnd-LED on] -

// - Yellow2 Status LED = Out4, J4:6, PD4 [high out-FET on to gnd-LED on]

// - FlashLight $=$ Out5, J4:7, PD5 [high out turns UVLED off \& FlashLight on] -

// - Analog +/- Power $=$ Out6, J4:8, PD6 [high turns on analog power] -

$/ / \quad$ - Latch Power On = Out7, J4:9, PD7 [high turns on FET latch main power] -

// - FlashLight Switch Yellow LED = OUT8, J4:10, PE2 [turn on LED] -

$/ /$ - Test Switch Yellow LED = OUT9, J4:11, PE3 [turn on LED]

$/ /$ - LCD BackLight $=$ PWM0, J2:10, PF4 $[100 \mathrm{~Hz} 50 \%$ duty cycle $]$ -

$/ /$ - On/Off Switch - Green LED = PWM1, J2:11, PF5 [100 Hz 50\% duty cycle] -

$/ /$ - Measure Switch - Red LED = PWM2, J2:12, PF6 [100 Hz 50\% duty cycle] -

// - K+ jumpered to VCC of LP3510 - R10 \& R12 = 0 ohms -

// - Detector signal acquired from serial A/D = LTC1864 -

// - Serial Port D operated in Clocked Synchronous mode -

// $\quad$ - Receive Data $=\mathrm{SRx}, \mathrm{PC1}, \mathrm{J6} 61$ [SDO on LTC1864] -

// $\quad$ - Sync Clock $=$ SCLK, PF0, J6:3 [SCK on LTC1864] -

// - Convert/ChipSelect $=$ SFCS, PB7, J6:8 [CONV on LTC1864] -

// $\quad$ - Transmit Data = STx, PC0, J6:6 [SDI not used on LTC1864] -

// - Synchronous Detected Signal acquired from serial A/D = LTC1864 -

// - Serial Port C operated in Clock Synchrouous mode - modify from RS232 -

// - Receive Data $=$ RxC, PC3, J4:21 moved JP3,2-3 [SDO on LTC1864]

// $\quad-$ Sync Clock $=$ TxE, J4:23, jump PF1 at U2pin17 to J4;23 [SCK LTC1864] -

// - Convert/CS = RxE, J4:24, jump PF3 at U2pin23 to J2:24 [CONV LTC1864] -

// - Transmit Data $=$ TxC, J4:20, PC2 moved JP4,2-3 [no SDI on LTC1864] -

$/ / \quad$ - Input Power $=\mathrm{J} 2: 16$ is 7 to 10 Volts

$/ / \quad$ - Ground $=\mathrm{J} 2: 17[+\mathrm{J} 2: 15, \mathrm{~J} 2: 13, \mathrm{~J} 2: 9 \mathrm{~J} 1: 9, \mathrm{~J} 6: 2, \mathrm{~J} 4: 1, \mathrm{~J} 4: 16, \mathrm{~J} 4: 25] \quad$ -

//-------------------------------------------------------------------------------------

$/ /$

$/ /$

void GetDataISR();

void StatusLEDs(char chVarSt);

void SwitchLEDs(char chVarSw);

int unStatus;

static int nReturnVal;

static unsigned int unPortDIn;

static char bPortDByteLo;

static char bPortDByteHi;

static unsigned int unPortCIn;

static char bPortCByteLo; 
static char bPortCByteHi;

static char bPowerOn; static char bBackGnd; static char bMeasure; static char bFlashLight; static char bTest; static int nDelayCount; static int nDisplayDLCounter; static long int lnActivityCounter;

static int nPowerSwitch; static int nPowerSource; static int nFlashLightSwitch; static int nMeasureSwitch; static int nTestSwitch; static char bMeasureClick; static char bFlashLightClick; static char bTestClick; static char bMeasurePushed; static char bFlashLightPushed; static char bTestPushed;

static unsigned long int freq;

static fontInfo fi6x8;

static windowFrame wfLCDtext;

const char BitRevLUT[] =

\{

0x00,0x80,0x40,0xC0,0x20,0xA0,0x60,0xE0,0x10,0x90,0x50,0xD0,0x30,0xB0,0x70,0xF0, 0x08,0x88,0x48,0xC8,0x28,0xA8,0x68,0xE8,0x18,0x98,0x58,0xD8,0x38,0xB8,0x78,0xF8, 0x04,0x84,0x44,0xC4,0x24,0xA4,0x64,0xE4,0x14,0x94,0x54,0xD4,0x34,0xB4,0x74,0xF4, $0 \mathrm{x} 0 \mathrm{C}, 0 \mathrm{x} 8 \mathrm{C}, 0 \mathrm{x} 4 \mathrm{C}, 0 \mathrm{xCC}, 0 \mathrm{x} 2 \mathrm{C}, 0 \mathrm{xAC}, 0 \mathrm{x} 6 \mathrm{C}, 0 \mathrm{xEC}, 0 \mathrm{x} 1 \mathrm{C}, 0 \mathrm{x} 9 \mathrm{C}, 0 \mathrm{x} 5 \mathrm{C}, 0 \mathrm{xDC}, 0 \mathrm{x} 3 \mathrm{C}, 0 \mathrm{xBC}, 0 \mathrm{x} 7 \mathrm{C}, 0 \mathrm{xF}$ $\mathrm{C}$, 0x02,0x82,0x42,0xC2,0x22,0xA2,0x62,0xE2,0x12,0x92,0x52,0xD2,0x32,0xB2,0x72,0xF2, 0x0A,0x8A,0x4A,0xCA,0x2A,0xAA,0x6A,0xEA,0x1A,0x9A,0x5A,0xDA,0x3A,0xBA,0x7A,0x FA, 0x06,0x86,0x46,0xC6,0x26,0xA6,0x66,0xE6,0x16,0x96,0x56,0xD6,0x36,0xB6,0x76,0xF6, 0x0E,0x8E,0x4E,0xCE,0x2E,0xAE,0x6E,0xEE,0x1E,0x9E,0x5E,0xDE,0x3E,0xBE,0x7E,0xFE, 0x01,0x81,0x41,0xC1,0x21,0xA1,0x61,0xE1,0x11,0x91,0x51,0xD1,0x31,0xB1,0x71,0xF1, 0x09,0x89,0x49,0xC9,0x29,0xA9,0x69,0xE9,0x19,0x99,0x59,0xD9,0x39,0xB9,0x79,0xF9, 0x05,0x85,0x45,0xC5,0x25,0xA5,0x65,0xE5,0x15,0x95,0x55,0xD5,0x35,0xB5,0x75,0xF5, 0x0D,0x8D,0x4D,0xCD,0x2D,0xAD,0x6D,0xED,0x1D,0x9D,0x5D,0xDD,0x3D,0xBD,0x7D,0x $\mathrm{FD}$, 0x03,0x83,0x43,0xC3,0x23,0xA3,0x63,0xE3,0x13,0x93,0x53,0xD3,0x33,0xB3,0x73,0xF3, 0x0B, 0x 8B,0x4B,0xCB,0x 2B,0xAB,0x6B,0xEB,0x1B,0x9B,0x5B,0xDB,0x3B,0xBB,0x7B,0xF $\mathrm{B}$, 0x07,0x87,0x47,0xC7,0x27,0xA7,0x67,0xE7,0x17,0x97,0x57,0xD7,0x37,0xB7,0x77,0xF7, $0 \mathrm{x} 0 \mathrm{~F}, 0 \mathrm{x} 8 \mathrm{~F}, 0 \mathrm{x} 4 \mathrm{~F}, 0 \mathrm{xCF}, 0 \mathrm{x} 2 \mathrm{~F}, 0 \mathrm{xAF}, 0 \mathrm{x} 6 \mathrm{~F}, 0 \mathrm{xEF}, 0 \mathrm{x} 1 \mathrm{~F}, 0 \mathrm{x} 9 \mathrm{~F}, 0 \mathrm{x} 5 \mathrm{~F}, 0 \mathrm{xDF}, 0 \mathrm{x} 3 \mathrm{~F}, 0 \mathrm{xBF}, 0 \mathrm{x} 7 \mathrm{~F}, 0 \mathrm{xFF}$ \} 
static char bNewData;

static int nIndex;

static unsigned int unDetectorAvg;

static unsigned int unSyncAvg;

static unsigned int unDetectorBack;

static unsigned int unSyncBack;

static int nDetectDiff;

static int nSyncDiff;

static unsigned long int ulnDetectorSum;

static unsigned long int ulnSyncSum;

static unsigned int unDetector[2048];

static unsigned int unSync[2048];

static char szSignalData[24];

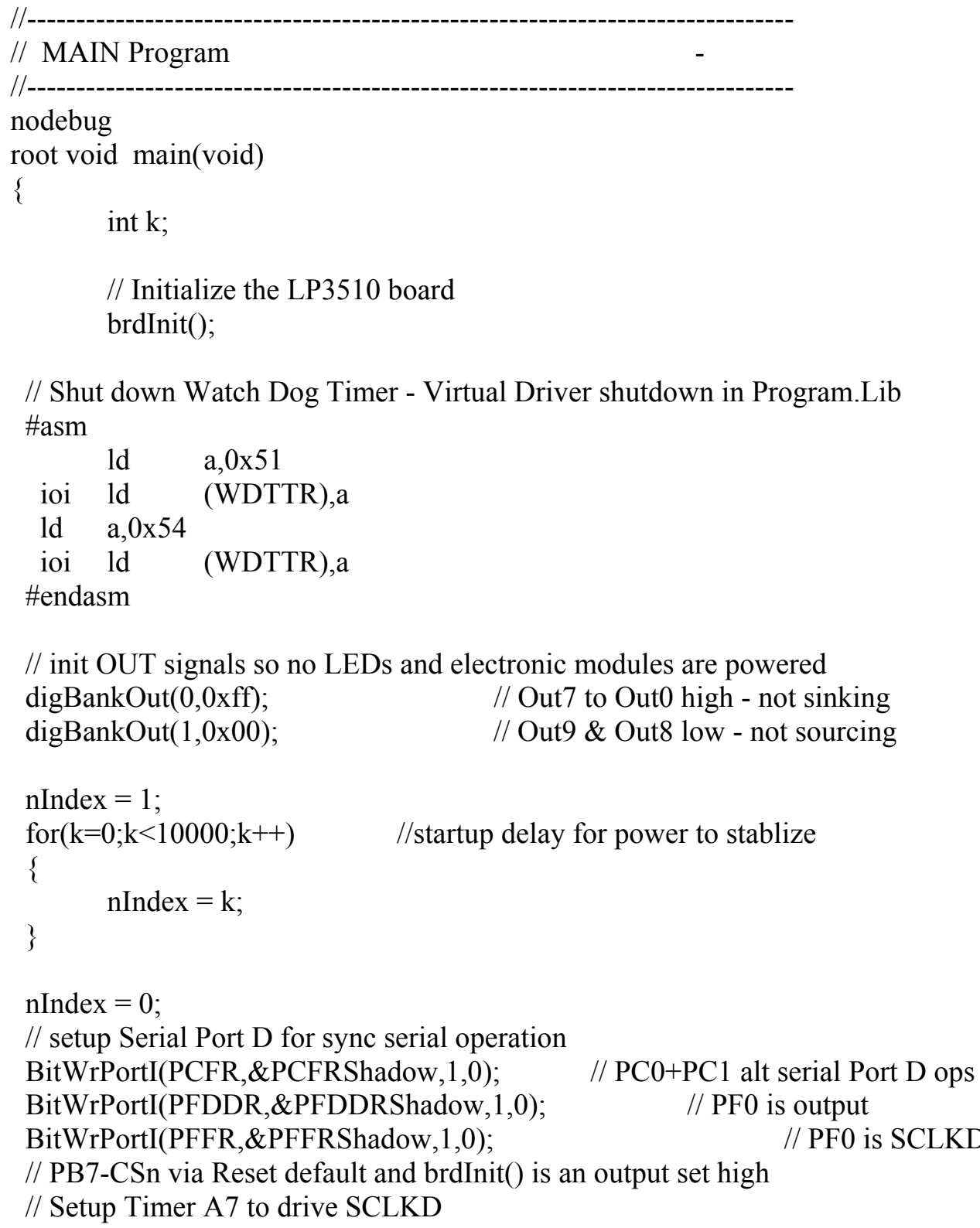


WrPortI(TAT7R,\&TAT7RShadow,1); $\quad$ // 2nd fastest clock - approx $1 \mathrm{MHz}$ // Setup Port D serial receive registers - Control and Extended

WrPortI(SDCR,\&SDCRShadow,0x0c); // no ops, recv enabled, clocked serial, no interrupts

WrPortI(SDER,\&SDERShadow,0x00); // no change - same as reset

// start Timer A7

BitWrPortI(TACSR,\&TACSRShadow,1,0);

// setup Serial Port C for sync serial opeation - hardware changes made to LP3510

BitWrPortI(PCFR,\&PCFRShadow, 1,2);

BitWrPortI(PFDDR,\&PFDDRShadow,1,1);

// PC2+PC3 alt serial Port C ops

BitWrPortI(PFFR,\&PFFRShadow,1,1);

BitWrPortI(PFDDR,\&PFDDRShadow,1,3);

// PF1 is output

// PF1 is SCLKC

BitWrPortI(PFDR,\&PFDRShadow,1,3);

// Setup Timer A6 to drive SCLK

WrPortI(TAT6R,\&TAT6RShadow,1);

// PF3 is output - CSn for Port C serial

// PF3 - CSn $=$ high

$/ /$ Setup Port C serial receive registers - Control and Extenced

WrPortI(SCCR,\&SCCRShadow,0x0c);// no ops, recv enabled, clocked serail, no interrupts

WrPortI(SCER,\&SCERShadow,0x00);

// same as when reset

// start Timer A6

BitWrPortI(TACSR,\&TACSRShadow,1,0);

// Setup Pulse Width Modulated Outputs

freq $=100 \mathrm{~L}$;

nReturnVal = pwmOutConfig(freq); $\quad$ // $100 \mathrm{~Hz}$ frequency

bPowerOn $=0$;

// Initialize Status

bBackGnd $=0$;

bMeasure $=0$;

bFlashLight $=0$;

bTest $=0$;

bNewData $=0$;

bMeasureClick $=0$;

bFlashLightClick $=0$;

bTestClick $=0$;

bMeasurePushed $=0$;

bFlashLightPushed $=0$;

bTestPushed $=0$;

for $(\mathrm{k}=0 ; \mathrm{k}<2048 ; \mathrm{k}++)$

// Initialize Data

\{

unDetector $[\mathrm{k}]=0$;

unSync $[\mathrm{k}]=0$;

\}

unPortDIn $=0$;

unPortCIn $=0$;

unDetectorAvg $=0$;

unSyncAvg = 0;

ulnDetectorSum $=0 \mathrm{~L}$;

ulnSyncSum = 0L; 


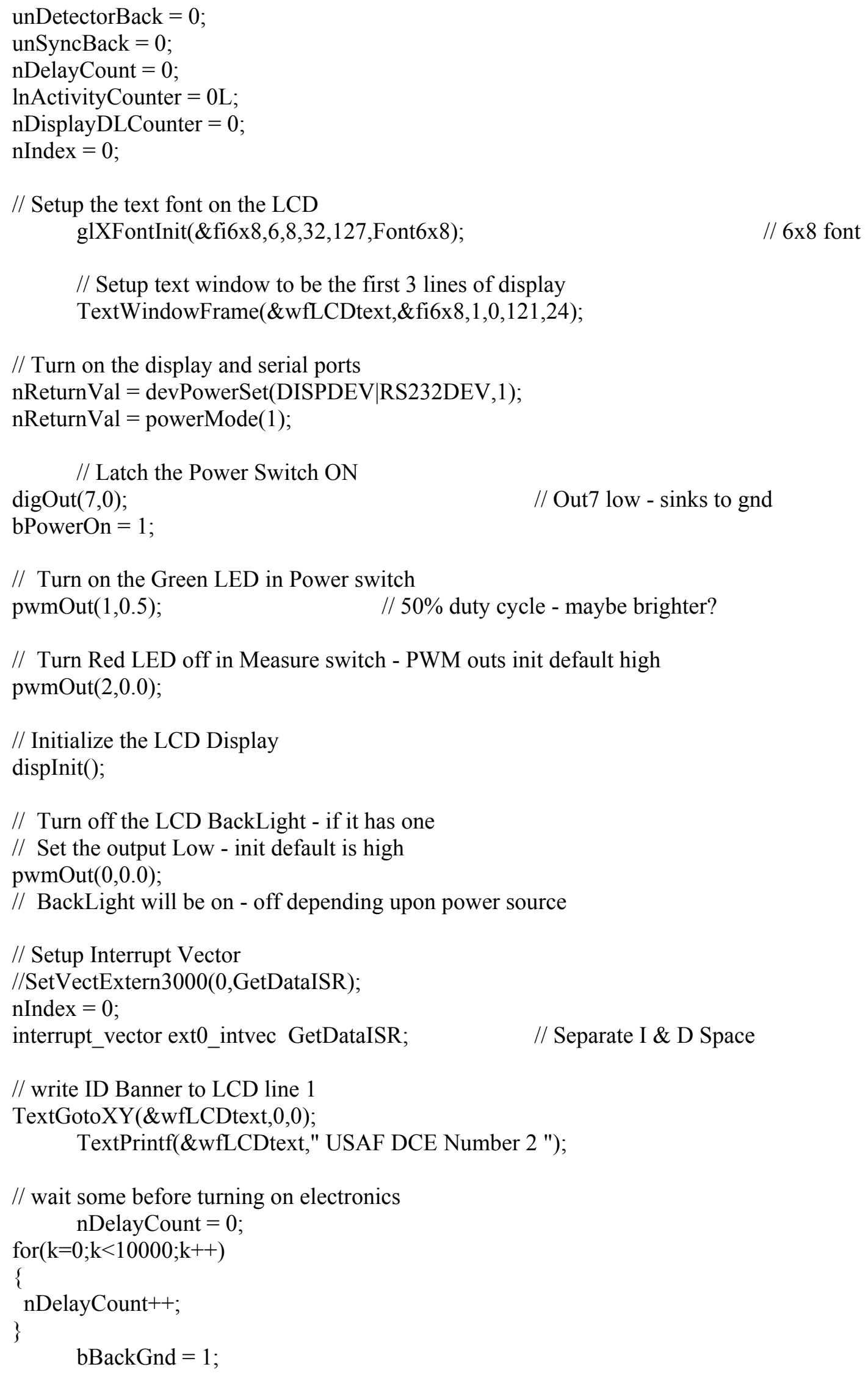




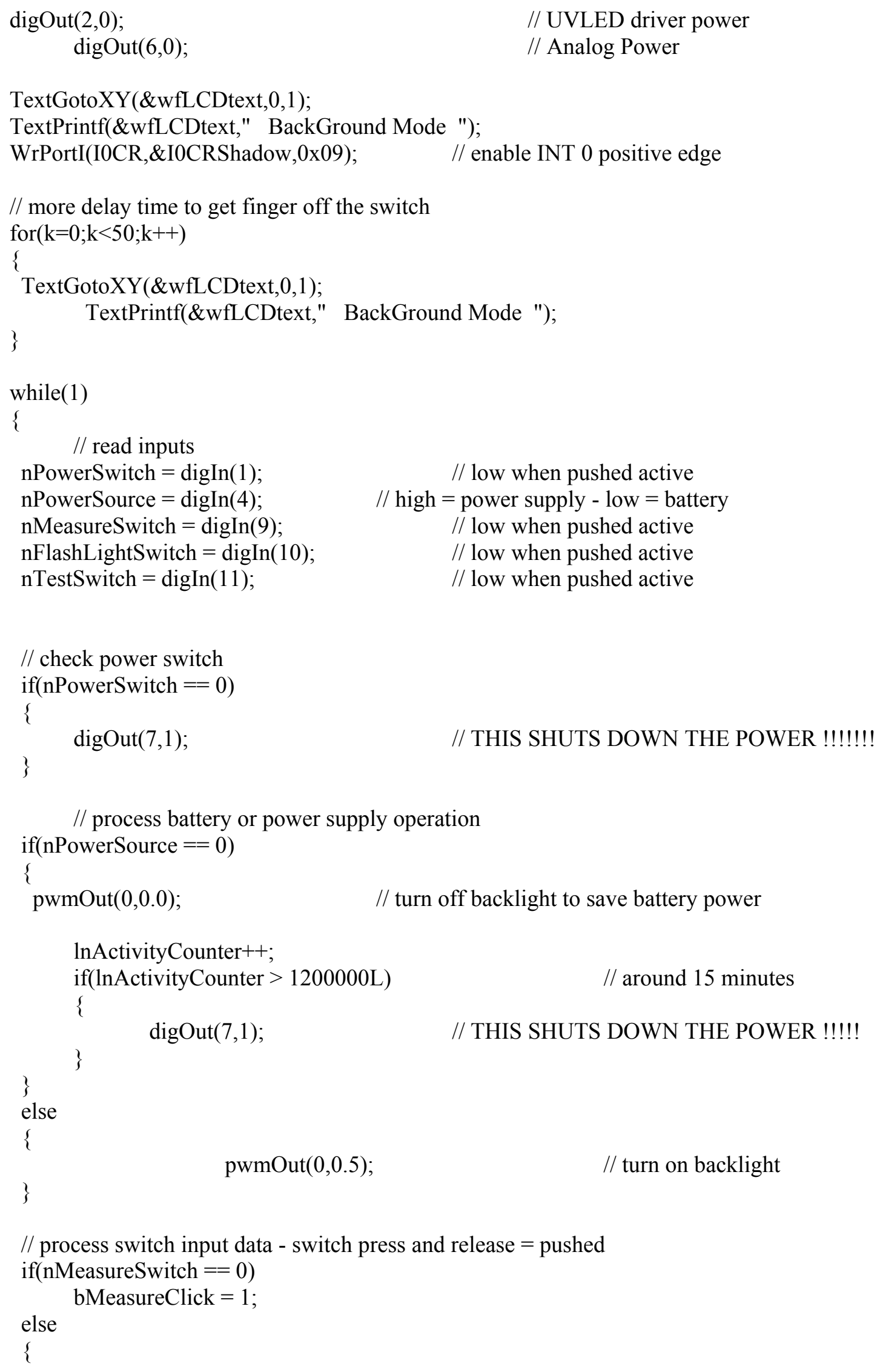




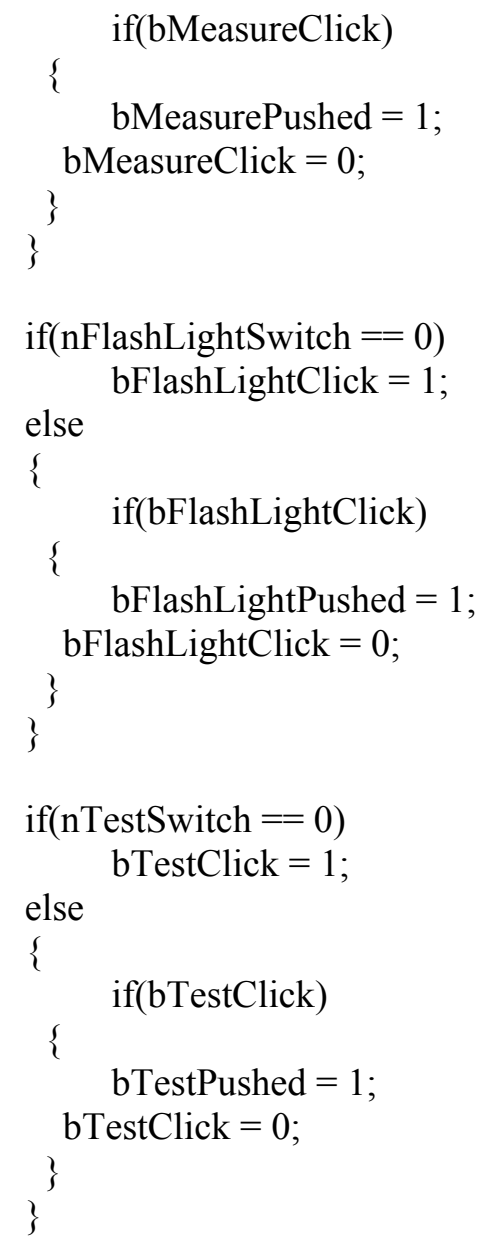




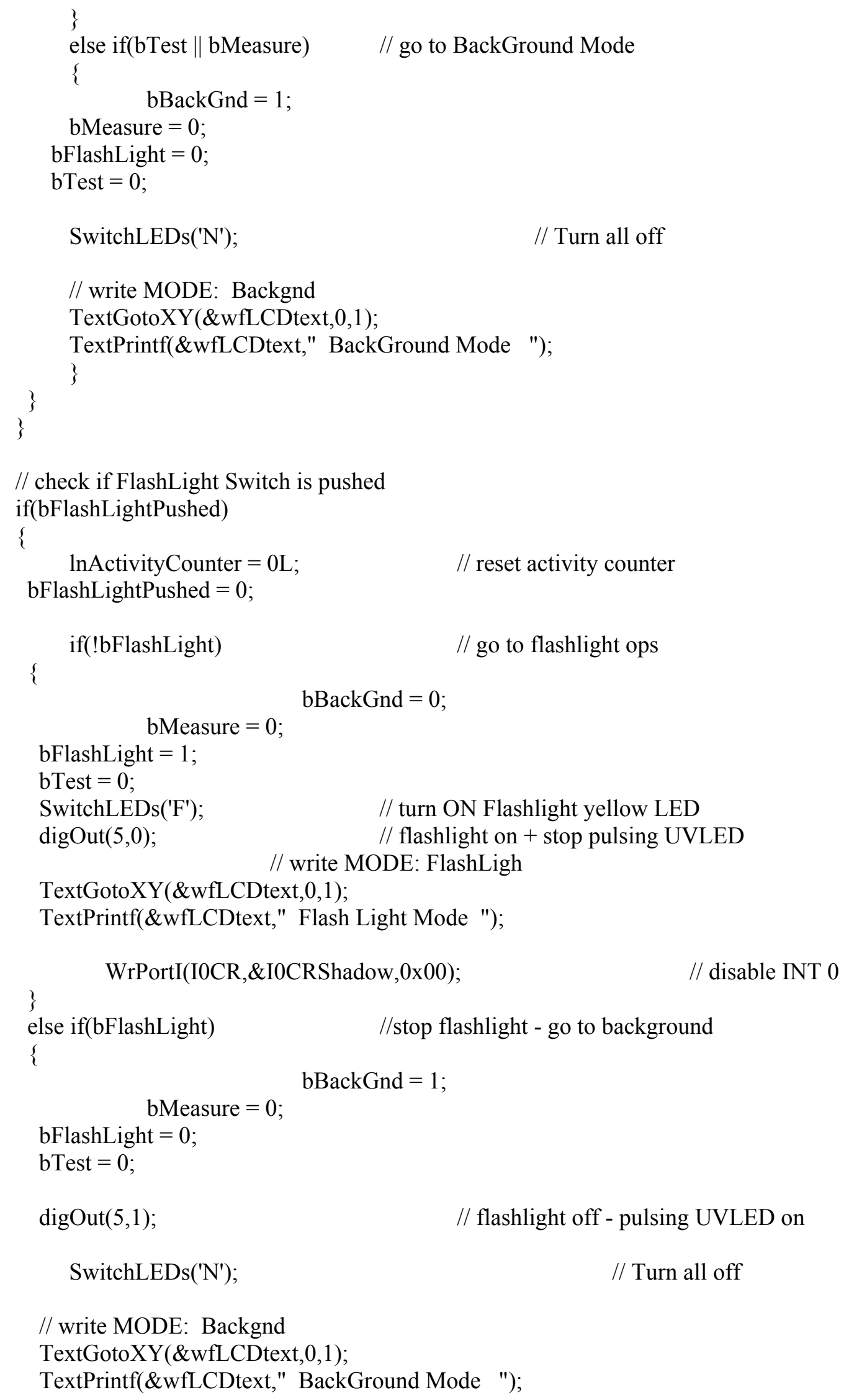




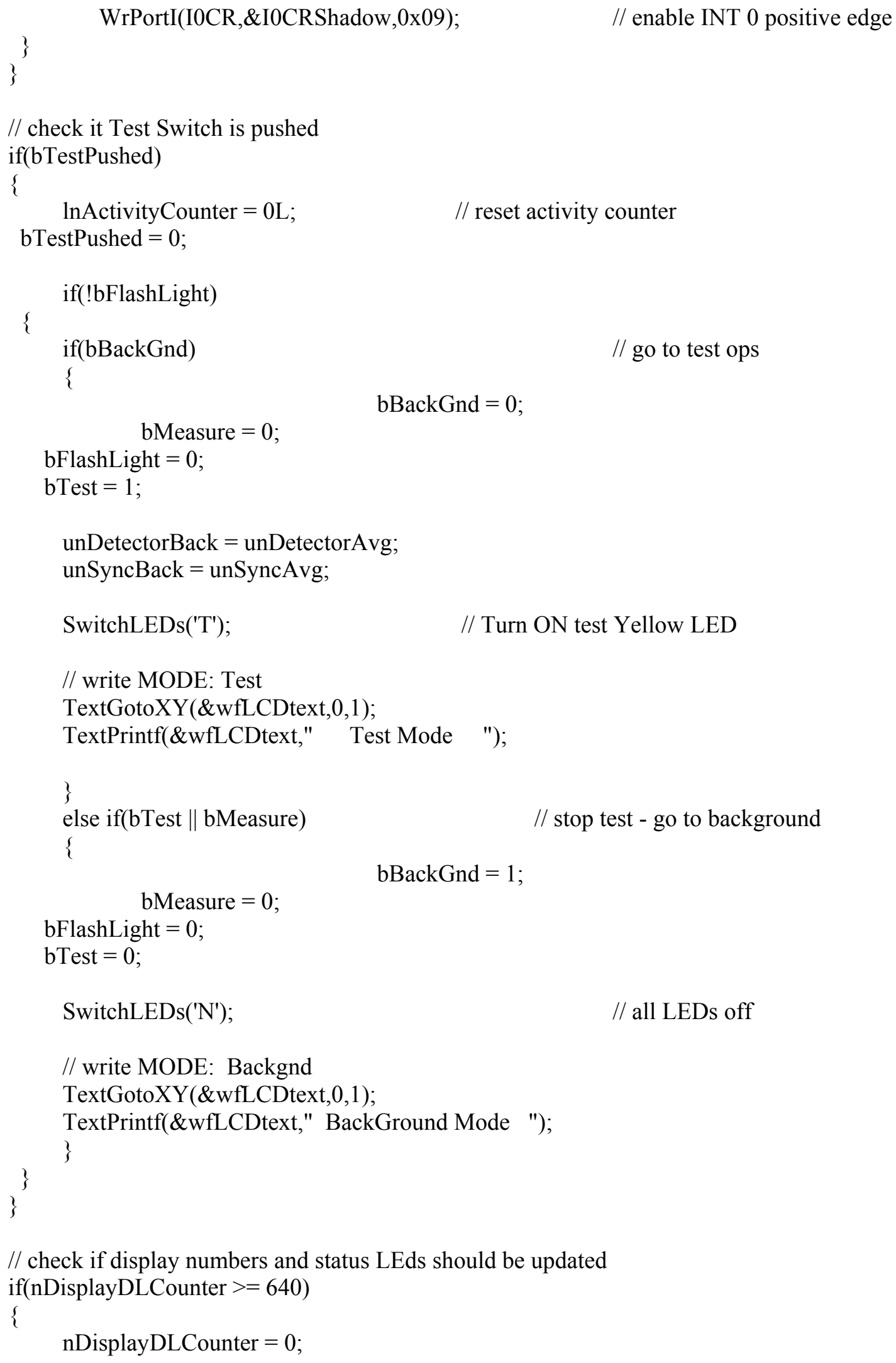




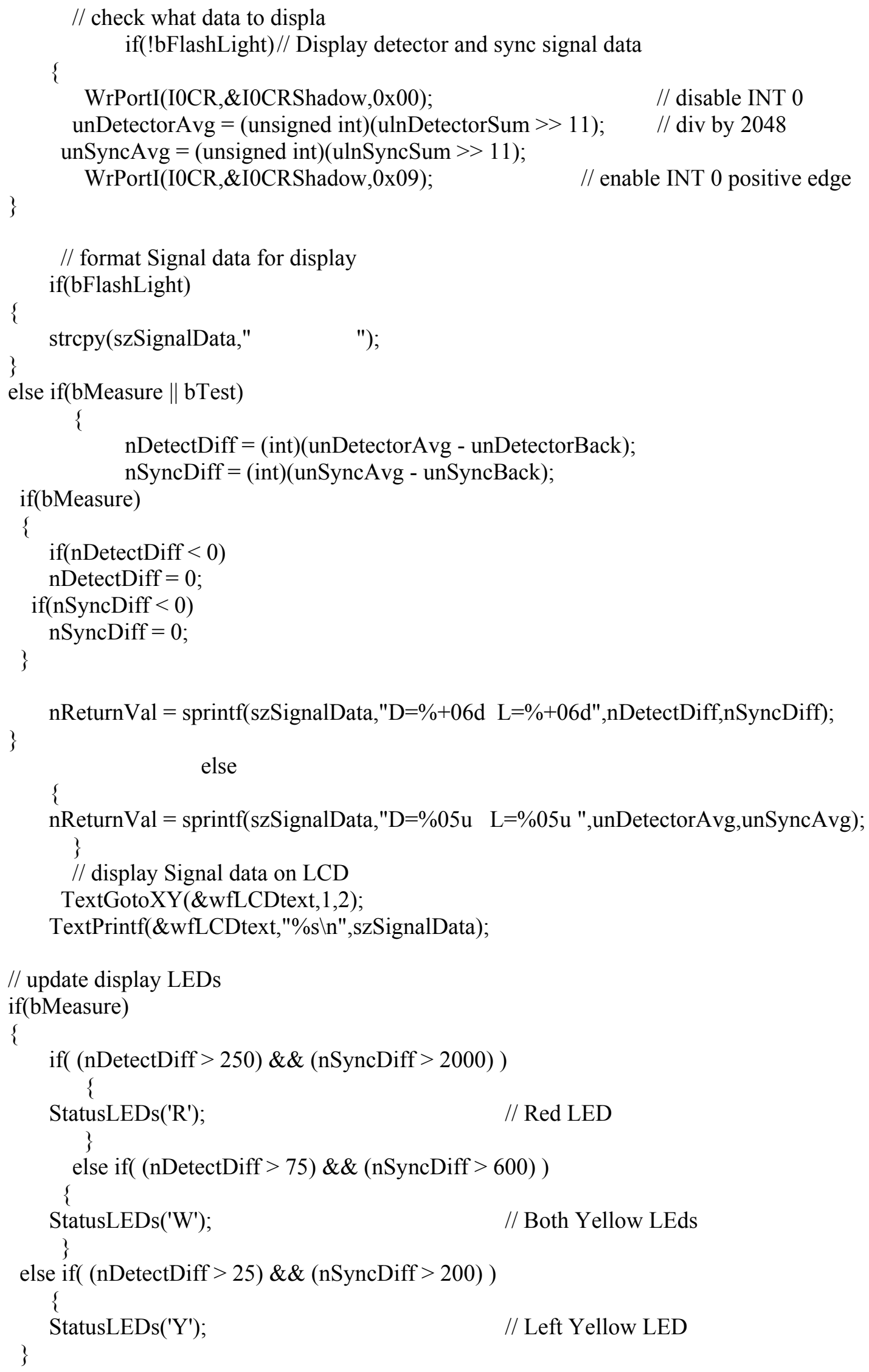




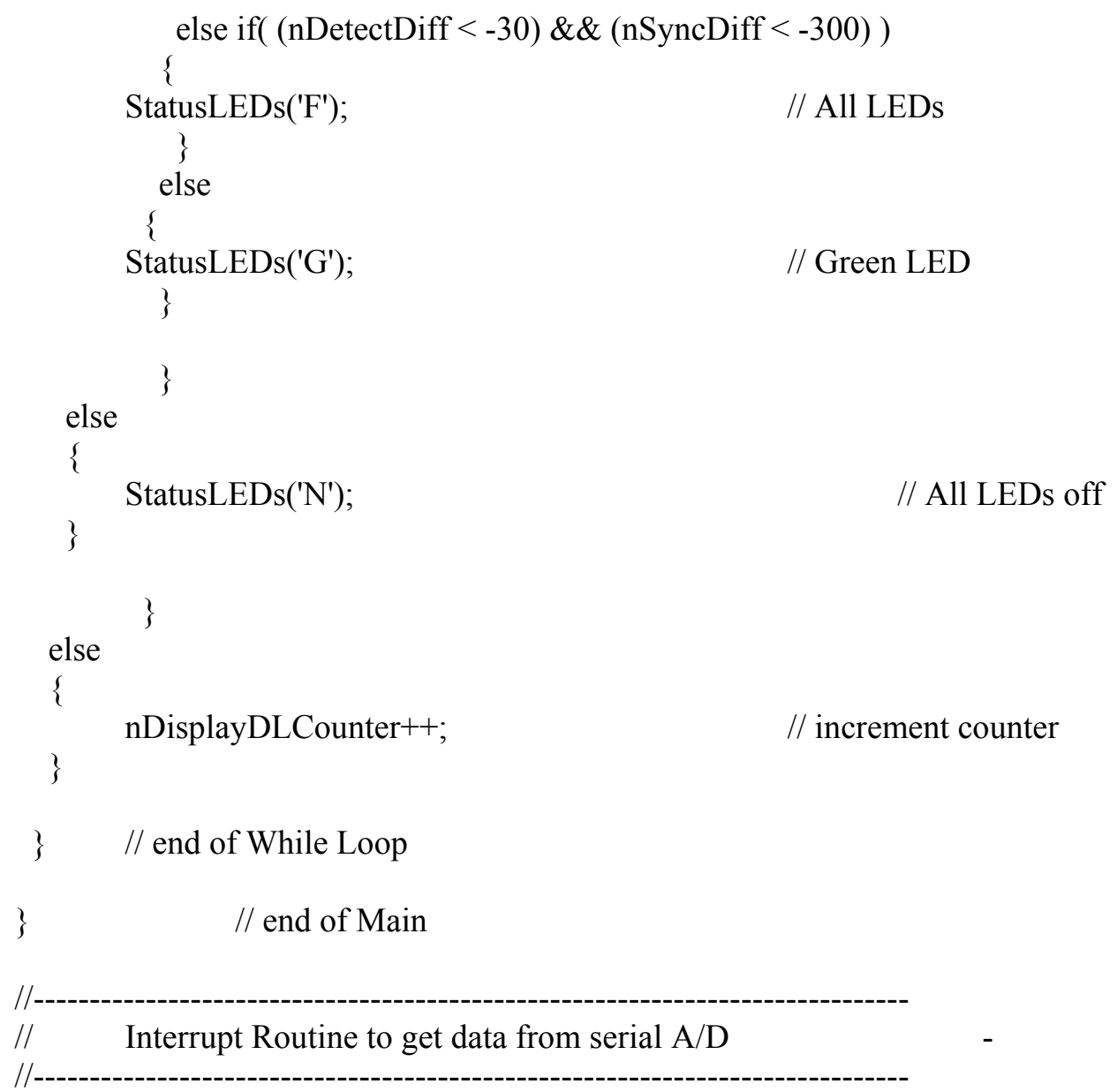




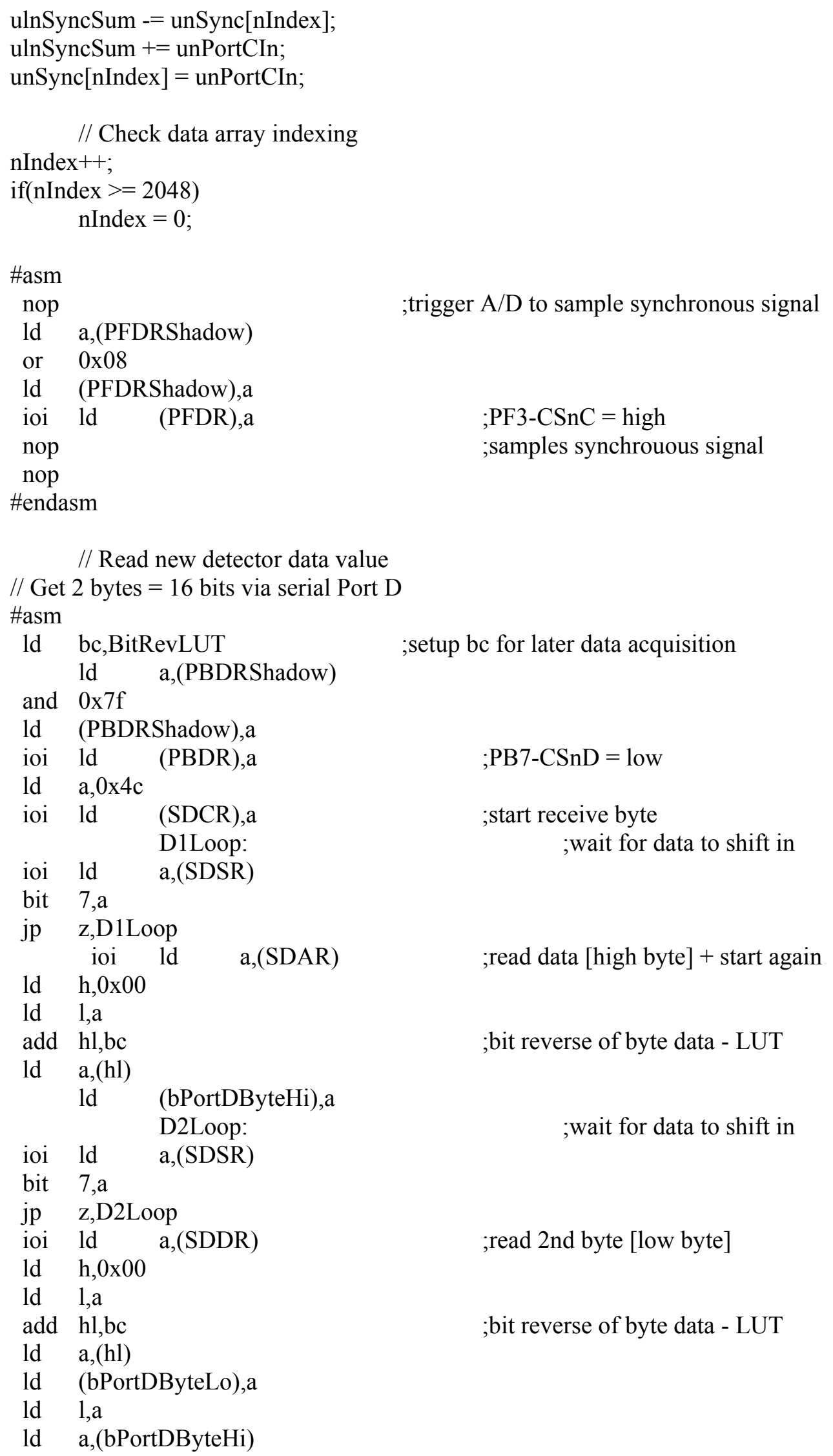




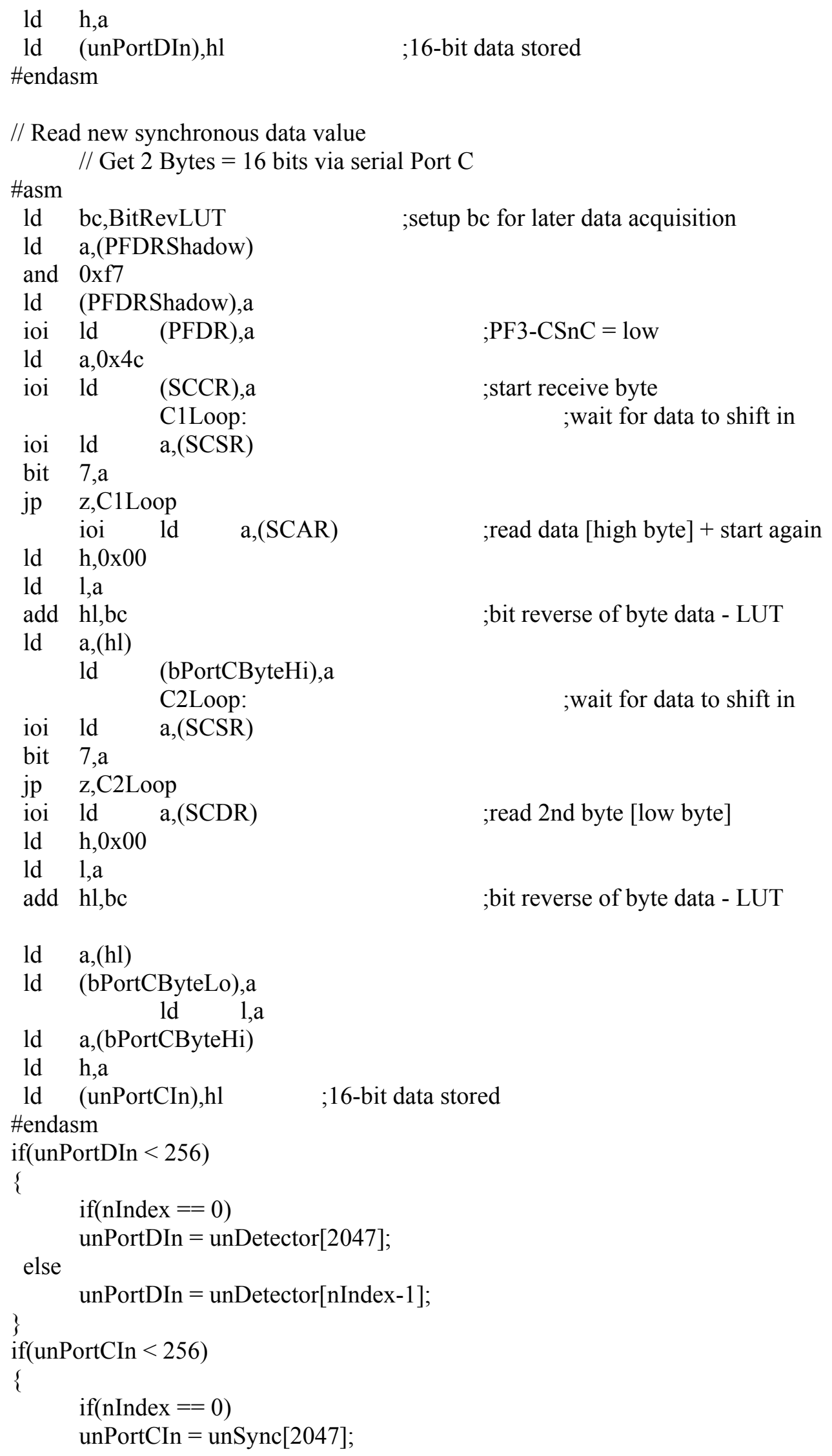




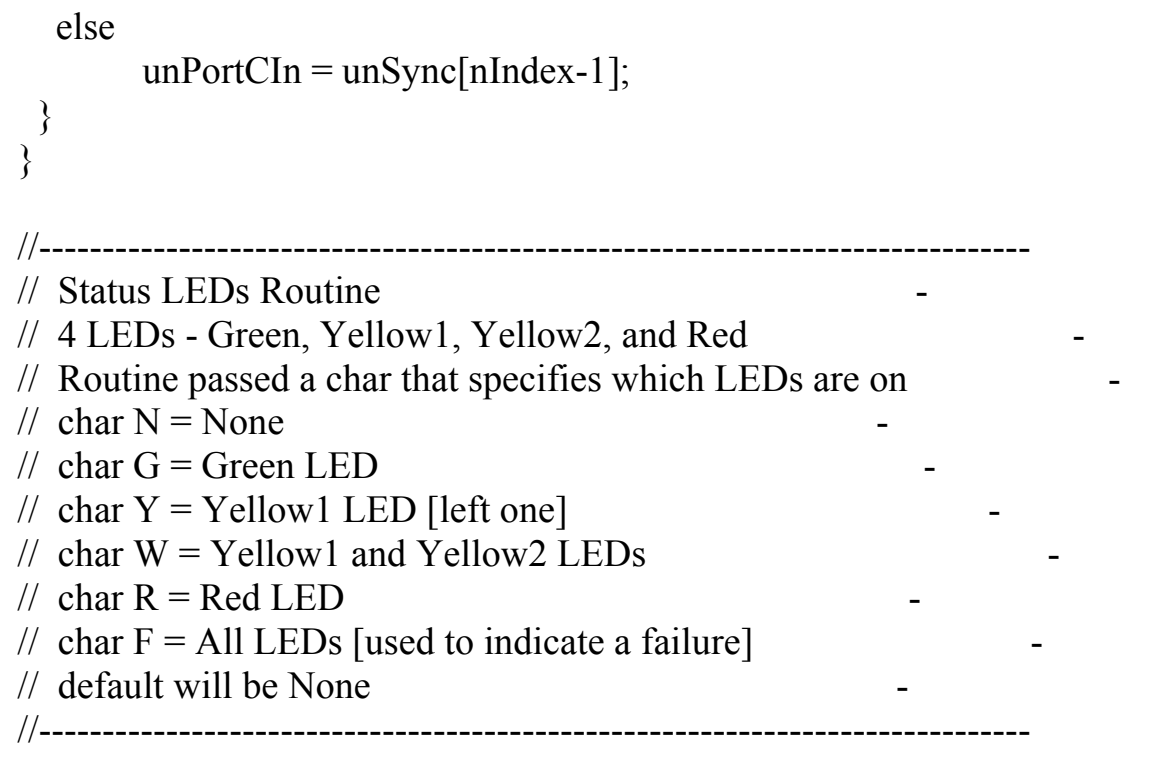

nodebug

root void StatusLEDs(char chVarSt)

\{

switch(chVarSt)

\{

case 'G':

$\begin{array}{ll}\operatorname{digOut}(0,0) ; & / / \text { Green }=\text { On } \\ \operatorname{digOut}(1,1) ; & / / \text { Red }=\text { Off } \\ \operatorname{digOut}(3,1) ; & / / \text { Yellow1 }=\text { Off } \\ \operatorname{digOut}(4,1) ; & / / \text { Yellow2 }=\text { Off }\end{array}$

break;

case 'R':

$\begin{array}{ll}\operatorname{digOut}(0,1) ; & / / \text { Green }=\text { Off } \\ \operatorname{digOut}(1,0) ; & / / \text { Red }=\text { On } \\ \operatorname{digOut}(3,1) ; & / / \text { Yellow1 }=\text { Off } \\ \operatorname{digOut}(4,1) ; & / / \text { Yellow2 }=\text { Off }\end{array}$

break;

case ' $\mathrm{Y}$ ':

break;

$\begin{array}{ll}\operatorname{digOut}(0,1) ; & / / \text { Green }=\text { Of } \\ \operatorname{digOut}(1,1) ; & / / \text { Red }=\text { Off } \\ \operatorname{digOut}(3,0) ; & / / \text { Yellow1 }=\text { On } \\ \operatorname{digOut}(4,1) ; & / / \text { Yellow2 }=\text { Off }\end{array}$

case 'W':

break;

$\begin{array}{ll}\text { digOut }(0,1) ; & / / \text { Green }=\text { Off } \\ \text { digOut }(1,1) ; & / / \text { Red }=\text { Off } \\ \text { digOut }(3,0) ; & / / \text { Yellow1 }=\text { On } \\ \operatorname{digOut}(4,0) ; & / / \text { Yellow2 }=\text { On }\end{array}$

case ' $F$ ':

$\begin{array}{ll}\operatorname{digOut}(0,0) ; & / / \text { Green }=\text { On } \\ \operatorname{digOut}(1,0) ; & / / \text { Red }=\text { On } \\ \operatorname{digOut}(3,0) ; & / / \text { Yellow1 }=\text { On }\end{array}$


break;

$\operatorname{digOut}(4,0) ; \quad$ // Yellow2 = On

case 'N':

default:

break;

$\begin{array}{ll}\operatorname{digOut}(0,1) ; & / / \text { Green }=\text { Off } \\ \operatorname{digOut}(1,1) ; & / / \text { Red }=\text { Off } \\ \operatorname{digOut}(3,1) ; & / / \text { Yellow1 = Off } \\ \operatorname{digOut}(4,1) ; & / / \text { Yellow2 }=\text { Off }\end{array}$

,

// Switch LEDs Routine

// 3 LEDs - Yellow, Yellow, and Red

// Routine passed a char that specifies which LEDs are on

// $\operatorname{char} \mathrm{N}=$ None

$/ /$ char $\mathrm{T}=$ Test Switch Yellow LED - uses digital output 9

$/ /$ char $\mathrm{F}=$ Flash Light Switch Yellow LED - uses digital output 8

// char $\mathrm{M}=$ Measure Switch Red LED - uses PWM output 2

// default will be None

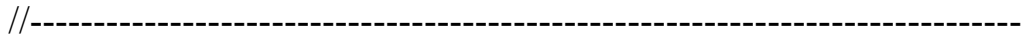

nodebug

root void SwitchLEDs(char chVarSw)

\{

switch(chVarSw)

\{

case 'M':

$\operatorname{pwmOut}(2,0.5)$;

$\operatorname{digOut}(8,0)$;

break;

$\operatorname{digOut}(9,0)$;

// Measure Red = On - 50\% duty cycle

$/ /$ Flash Yellow $=$ Off

$/ /$ Test Yellow $=$ Off

case 'F':

break;

case ' $\mathrm{T}$ ':

$\operatorname{pwmOut}(2,0.0)$;

$\operatorname{digOut}(8,1)$;

$\operatorname{digOut}(9,0)$;

// Measure Red $=$ Off

// Flash Yellow $=$ On

$/ /$ Test Yellow $=$ Off

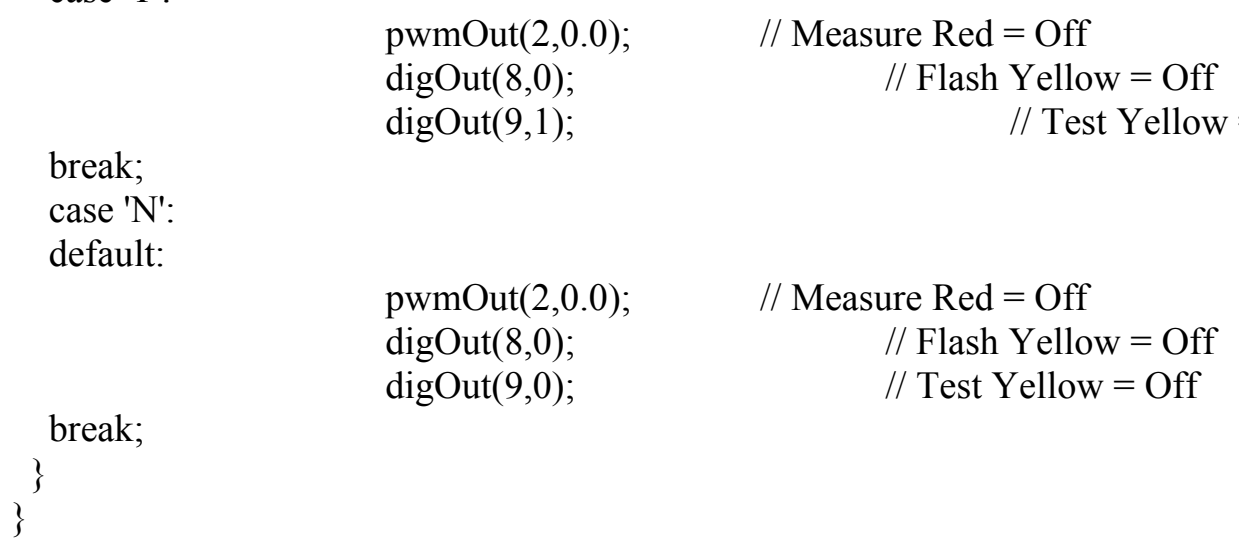




\section{Cartridge Design}

The concentrating cartridge design is driven by the following considerations:

- Large enough to hold comfortably with gloved hands

- Vacuum sealed for ease of filling via syringe through a rubberized septum

- Able to accommodate and concentrate at 500 micoliter sample volume

- Integral magnet to allow filling and concentration in the absence of the DCE instrument

- Rugged enough to resist drop fracture or separation

- Stable enough to withstand external decontamination via bleach solution without compromising the contents.

- Long term sealed for chain of custody considerations.

Photographs of the assembled cartridges are shown in Figure 15. The photographs show close up views of the concentration magnet and the fill septum.
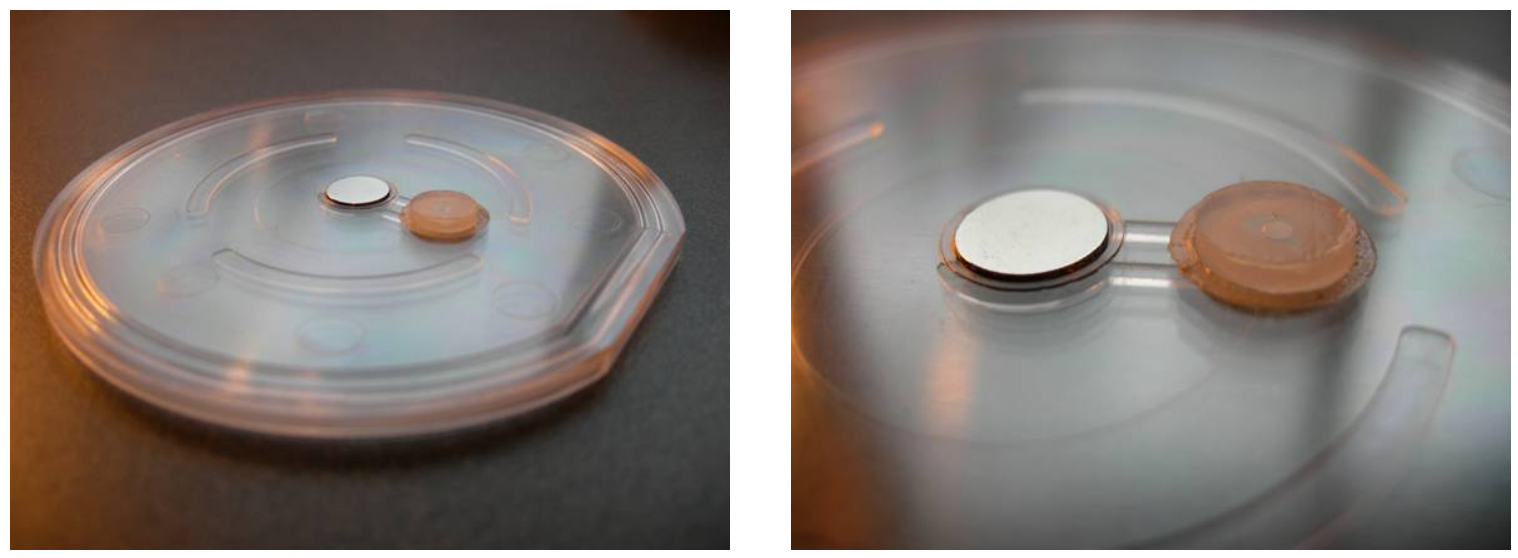

Figure 15. Photographs of assembled DCE cartridge including close up of magnet and injection septum.

Dimensioned drawings of the cartridge assembly are shown in Figures 16-18. The main body of the cartridge is an injection molded transparent polycarbonate construction. The main body is ultrasonically welded to a cover sheet of clear polycarbonate forming a 500 micoliter cavity with a guided fluid channel so that all material injected flows past the embedded magnet.

The two piece cartridge bodies are molded and ultrasonic welded by Plastic Injection Molding, Inc (PMI), Richland, WA 509-375-4260. The molds and the welding tooling have been retained in storage by PMI for future fabrication or client disposition at a later date. 


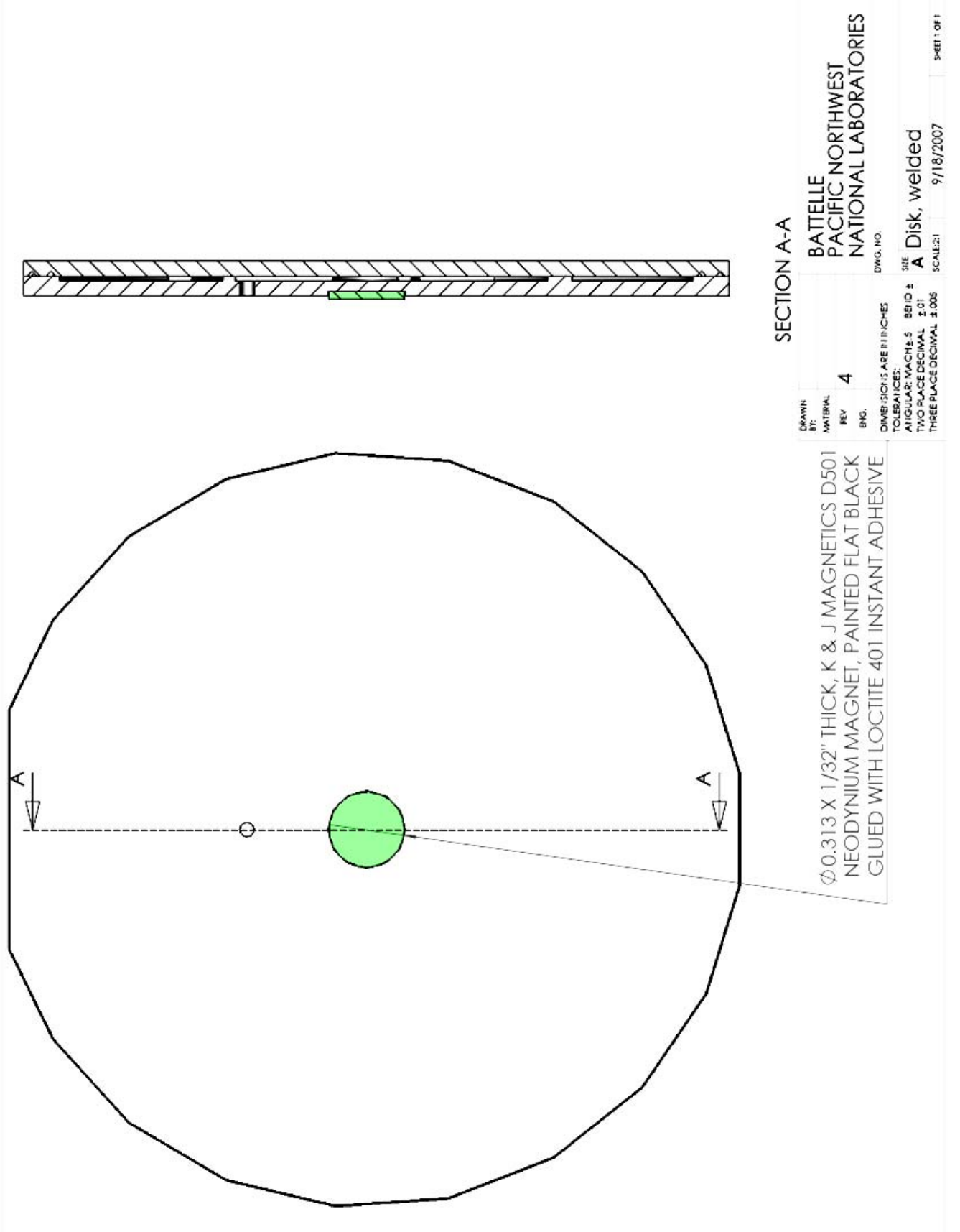

Figure 16. Dimensional drawing of DCE cartridge assembly. 


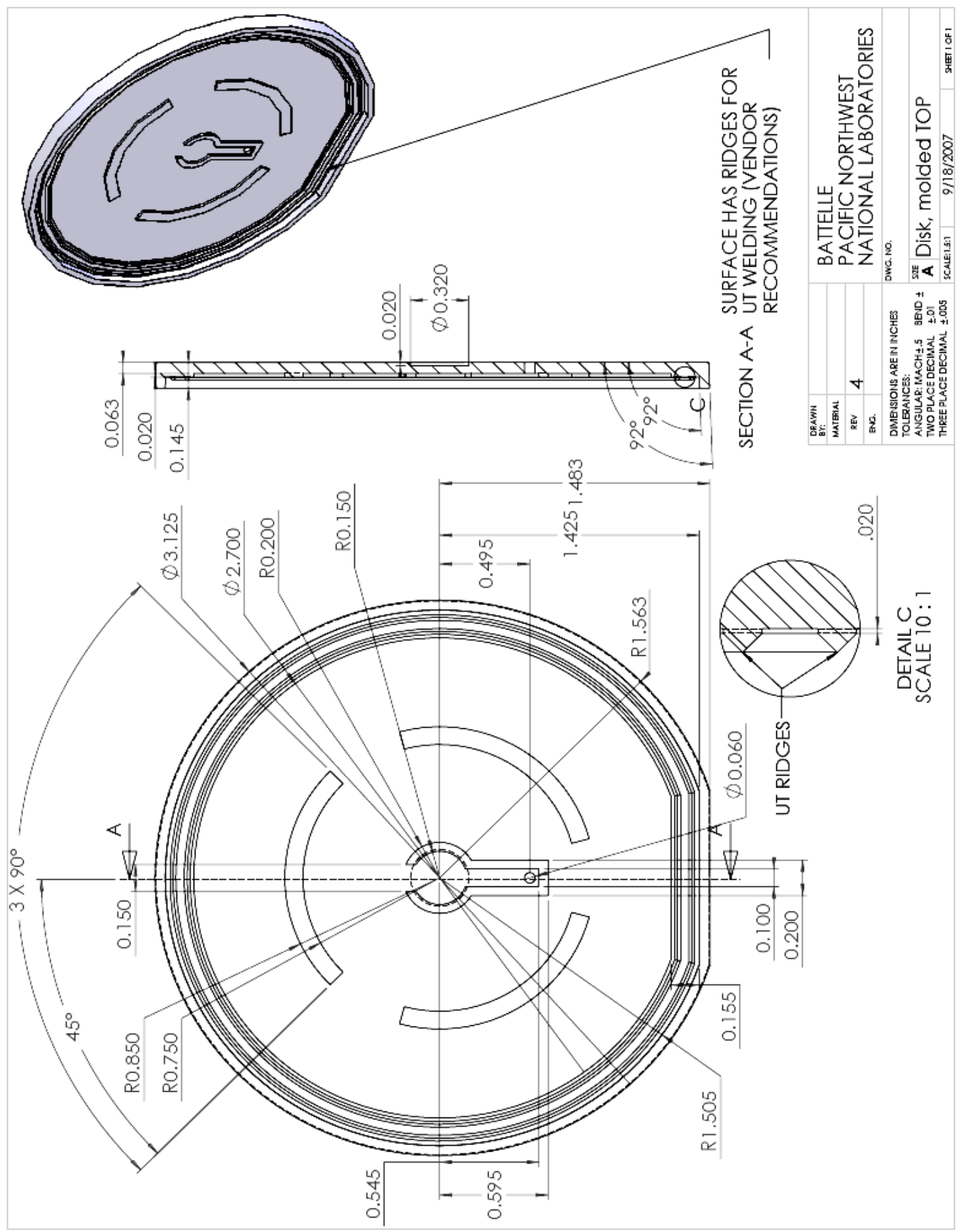

Figure 17. Dimensional drawing of DCE cartridge molded top detail.

37 

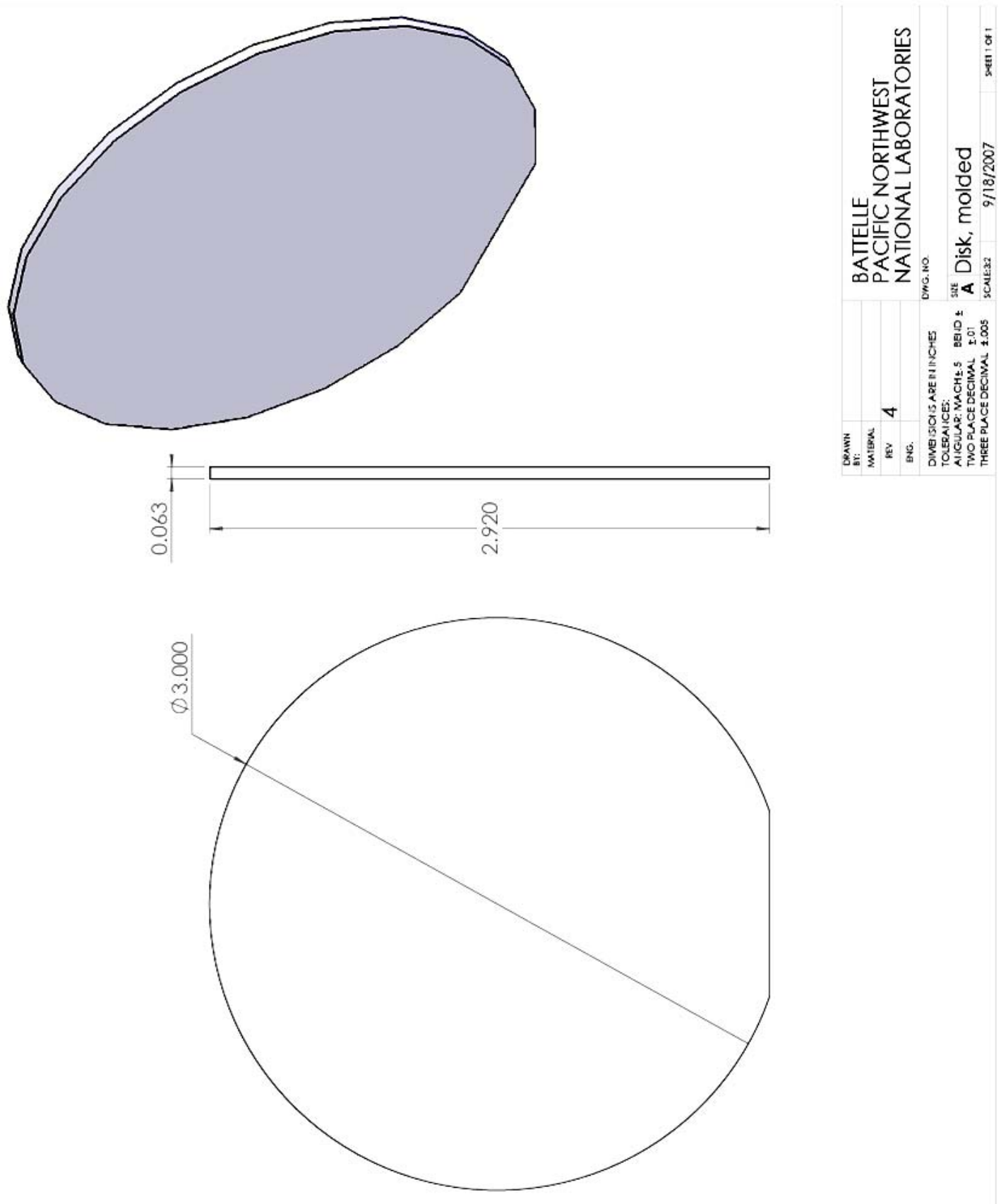

Figure 18. DCE cartridge bottom detail. 


\section{Process notes on cartridge assembly:}

The cartridges are outgassed by overnight baking at $95 \mathrm{C}$ and 20 " $\mathrm{Hg}$ vacuum. The prototype were outgases at PNNL.

Prepainted (black) $0.313 \times 0.0313$ neodymium disk magnets (D501, K\&J Magnetics) are affixed to the exterior center recess using Loctite 401 adhesive. These magnet disks attract and concentrate the magnetic beads for the optical assay when the alalyte stream is injected into the cartridge.

$8.0 \mathrm{~mm}$ diameter injection port septa are cut from sheet stock of .050 " - 50 durometer silicone bonded to .002" FEP Teflon. (part ST58/2, Specialty Silicone, 518-885-8826). The dual material provides a lower air diffusion profile.

The septa are affixed to the cartridge by applying a ring of polyurethane adhesive sealant (3M Scotch Seal 560 Gray) concentric with the cartridge fill port, then affixing each septum with the Teflon side up.

After $>2$ hours cure time the cartridges are evacuated by puncturing each septum with a 30 gauge needle plumbed to 22 " $\mathrm{Hg}$ vacuum. In the prototype production run about $10 \%$ seal failure occurred after seal puncture via leakage through the cartridge perimeter. Groups of four cartridges oriented back-to-back are immediately sealed in bags using a Food Saver V2220 vacuum sealer for distribution and shipment.

\section{Notes on cartridge testing:}

Limited initial testing was performed on the assembled cartridges. After 25 days two bags were opened to test vacuum and the water draw capacity of the eight cartridges. The vacuum in the bags at this time was greater 10.3" Hg. Actual vacuum levels are likely higher than reported by 1 to 2 in $\mathrm{Hg}$ as the measurement process injects a small amount of air at atmospheric pressure before each measurement.

Water draw tests were conducted by filling a $3 \mathrm{ml}$ syringe with a 30 gauge needle to 900 $\mu \mathrm{l}$ with no plunger in the syringe. The open syringe was used to puncture the septum and 5 minutes was allowed for flow, then the volume change at the syringe was noted. Final flows varied from $350-900 \mu \mathrm{l}$ and took between 22 and five minutes depending the insertion technique.

Because of the insertion variability, a simple puncture jig would make an excellent accessory to insure minimum initial evacuation failure and maximum low and minimum fill times during cartridge operation. 